\title{
Compensatory Paracrine Mechanisms That Define The Urothelial Response to Injury in Partial Bladder Outlet Obstruction
}

Thomas S. Lendvay ${ }^{1,6}$, Robert Sweet ${ }^{2}$, Chang-Hee Han ${ }^{1}$, Tarkan Soygur ${ }^{1}$, Jan-Fang Cheng ${ }^{3}$, J. Chadwick Plaire ${ }^{1}$, Jay S. Charleston ${ }^{4}$, Lynne B. Charleston ${ }^{4}$, Shelly Bagai ${ }^{1}$, Kimber Cochrane ${ }^{1}$, Eric Rubio $^{1}$, Elaine Fuchs ${ }^{5}$ and James A. Bassuk ${ }^{1,2}$

From the ${ }^{1}$ Program in Human Urothelial Biology, Children's Hospital and Regional Medical Center, Seattle, WA; ${ }^{2}$ Department of Urology, University of Washington School of Medicine,

Seattle, WA; ${ }^{3}$ Department of Genome Sciences, Ernst Orlando Lawrence Berkeley Laboratory, Berkeley, CA; ${ }^{4}$ Stereotome Northwest, Issaquah, WA; ${ }^{5}$ Laboratory of Mammalian Cell Biology and Development, Howard Hughes Medical Institute, The Rockefeller University, New York, NY

Running Title: Compensation by FGF-10 in FGF-7 Null Mice

Keywords: bladder, urothelial, transitional epithelium, fibroblast growth factor -7 , fibroblast growth factor-10, compensation keratinocyte growth factor

${ }^{6}$ Correspondence: Thomas Lendvay, M.D.

Department of Surgery, MailStop W-7729

Children's Hospital and Regional Medical Center 4800 Sand Point Way NE

Seattle, WA 98105

thomas.lendvay@seattlechildrens.org

206-987-1270 (office), 206-987-4220 (fax) 


\begin{abstract}
Diseases and conditions affecting the lower urinary tract are a leading cause of dysfunctional sexual health, incontinence, infection and kidney failure. The growth, differentiation, and repair of the bladder's epithelial lining is regulated by fibroblast growth factor (FGF)-7 and -10 via a paracrine cascade originating in the mesenchyme (lamina propria) and targeting the receptor for FGF-7 and 10 within the transitional epithelium (urothelium). Recombinant FGF- 7 and -10 induce proliferation of human urothelial cells in vitro and transitional epithelium of wild-type and FGF7null mice in vivo. To determine the extent that induction of urothelial cell proliferation during the bladder response to injury is dependent on FGF-7, an animal model of partial bladder outlet obstruction was developed. Unbiased stereology was used to measure the percentage of proliferating urothelial cells between obstructed groups of wild-type and FGF7-null mice. The stereological analysis indicated that a statistical significant difference did not exist between the two groups, suggesting that FGF-7 is not essential for urothelial cell proliferation in response partial outlet obstruction. In contrast, a significant increase in FGF-10 expression was observed in the obstructed FGF-7 null group, indicating that the compensatory pathway that functions in this model result in urothelial repair.
\end{abstract}




\section{INTRODUCTION}

Bladder outlet obstruction is a medical condition that can affect men, women and children. Lower urinary tract obstruction can lead to voiding dysfunction as well as kidney failure. Many men suffer from obstruction due to benign prostatic hypertrophy, leading to both medical and psychosocial dysfunction (2). Associated with these dysfunctions are severe losses of smooth muscle function and bladder contractility, or progression of the bladder towards conditions of severe hypertrophy and hypercontraction (3). Incomplete bladder emptying in patients is also a risk for urinary tract infection. In addition, children born with congenital forms of bladder outlet obstruction such as posterior urethral valves or children born with functional bladder obstruction, such as in spina bifida patients, can lead to significant urinary incontinence and renal dysfunction.

Transitional epithelium that lines the urinary tract exhibits one of the slowest turnover rates among mammalian epithelia, estimated at once every 365 days (4-7). Although the urothelial cells that comprise this epithelia rest in $\mathrm{G}_{0}$ during normal conditions, they remain active in metabolic processes that accompany shape change during bladder emptying and filling. In response to injury, transitional epithelium exhibits a remarkable ability to turnover within $24-48$ hours after the initial insult. The identity of the mitogenic signal that gives rise to basal urothelial cell proliferation has been attributable to growth factors. Such factors are classified as either paracrine, autocrine, or juxtacrine, depending on the originating and target cell type. Mitogens known to act on human urothelial cells include epidermal growth factor (8), heparin-binding epidermal growth factor-like growth factor (9), activators of the estrogen receptor $(10,11)$, transforming growth factor- $\alpha(12)$, fibroblast growth factor-1 (12), and fibroblast growth factor-10 (13).

The model most frequently used to study the urothelial response to injury is partial outlet obstruction of the bladder via urethral ligation (14). The wound healing response appears to be constant from animal model to animal model and mimics the clinical response seen in humans (15- 
17). Such events include urothelial cell proliferation within $24-48 \mathrm{hr}$, subsequent hypertrophy of the bladder wall, and alterations in detrusor muscle and the extracellular matrix. Experimental animal models have been exploited to study bladder dysfunctions that are similar to those observed in men with benign prostatic hyperplasia $(18,19)$. Furthermore, this experimental model has direct implications for other clinical disease states such as congenital (i.e. posterior urethral valves) or acquired (urethral stricture or bladder neck contraction) urinary tract obstruction.

Fibroblast growth factor-7 (FGF-7) is a 194 aa polypeptide implicated in both the induction of basal urothelial cell proliferation and the expansion of transitional epithelium $(14,20)$. Also known as keratinocyte growth factor $(21,22)$, FGF-7 is one of 24 members of the FGF family of polypeptide growth factors. The crystal structure of FGF-7 has been solved $(1,23)$. The protein folds into a beta-trifoil motif similar to other members of the FGF family whose structures have been solved. Although FGFs 11-14 exhibit striking structural similarities to FGF-10, FGFs 11-14 have diverged to direct related surfaces towards interaction with protein targets distinct from canonical FGF receptors (FGFR) (24).

FGF-7 and FGF-10 are considered to be paracrine factors, originating in mesenchyme but active only upon epithelium $(13,21,22,25)$. The formation of a specific FGF7-containing FGFR signal transduction complex requires 3 components: the FGF-7 polypeptide, proteoglycans that contain either heparan- $(26,27)$, dermatin- (28), or chondroitin- (29) sulfate, and an alternatively-spliced tyrosine kinase protein product of the FGFR2 gene $(7,30)$, and (27). This receptor, known as the keratinocyte growth factor receptor (KGFR), the FGFR2IIIb splice variant, or the FGFR2 isoform 2 splice variant, is a cell-surface transmembrane protein that undergoes dimerization upon ligand binding and subsequent autophosphorylation of intracellular tyrosine residues (31). Allelic mutations in the FGFR2 exons that encode the isoform 2 splice variant are known to give rise to a 
number birth defects that include craniosynostosis syndromes (32). It is believed that the FGFR2 isoform 2 splice variant serves as a receptor for both FGF-7 and FGF-10.

Mice that contain a targeted disruption of the FGF-7 gene exhibit defects in (i) cells that give rise to the hair shaft (33), (ii) ureteric bud outgrowth (34), and (iii) stratification of bladder urothelium (35). The collective evidence demonstrates an essential role for FGF-7 in development, growth, differentiation, and homeostasis of the mucosal lining of the urinary tract. In contrast to the FGF-7 null mouse, the FGF-10 null mouse lacks limb bud initiation and lung development (36). In addition, the FGF-10 null mouse exhibits gross anatomical defects in the urinary tract with includinga urothelium that fails to stratify (37). Consequently, FGF-10 null mice die at birth, precluding their use in experimental models of partial bladder obstruction.

This study demonstrated the mitogenic potential of FGF-7 on human and murine urothelial cells. The growth factor was found to stimulate resting cells to exit from $\mathrm{G}_{0}$ and traverse the cell cycle. To determine if the mitogen was responsible for the induction of urothelial cell proliferation during the initial response of the bladder to injury, we examined whether there was a difference in the urothelial response to injury between wild-type and FGF7-null mice in a model of partial outlet obstruction. Stereological analysis of obstructed bladders revealed that a statistically significant difference did not exist indicating that FGF-7 is not essential for urothelial cell proliferation during the response to partial outlet obstruction of the urinary bladder. Further stereological analysis revealed a significant fold increase of FGF-10 synthesis in obstructed FGF7-null mice via a compensatory paracrine mechanism. 


\section{RESULTS}

\section{Isolation and Sequence Analysis of FGF-7 cDNA from Human Urinary Bladder}

A $0.492 \mathrm{kbp}$ partial cDNA fragment encoding FGF-7 from a human urinary bladder cDNA library by reverse transcription and PCR was cloned, sequenced, and found to be $100 \%$ identical with the corresponding region present in the NCBI reference sequence encoding human lung FGF-7 mRNA (Accession NM_002009.2). Analysis of the $0.492 \mathrm{kbp}$ bladder FGF-7 sequence predicted an open reading frame for a polypeptide exhibiting a length of 164 residues, a $\mathrm{M}_{\mathrm{r}}$ of 18,594.70, a pI of 9.82, and a molar extinction coefficient of $21,770 \mathrm{~mol} \mathrm{~g}^{-1} \mathrm{~cm}^{-1}$. These data are consistent with the deduced primary structure being the mature, secreted form of bladder FGF-7.

\section{Distribution and Characterization of FGF-7 Sequences in the Human Genome}

The distribution of the bladder FGF-7 cDNA sequence in the human genome is shown in Table 1. Regions of identity or near-identity were localized to chromosomes $9,15,18$, and 21 . Because our bladder FGF-7 cDNA sequence does not contain 5'- and 3'- mRNA sequences, we performed an analogous search with the reference sequence assigned to the FGF-7 mRNA by the National Center for Biotechnology Information. In Table 1, the bracketed row of data represents additional genomic regions detected with the full-length 3,853 base mRNA reference sequence. Five, four, two and two regions of identity or near-identity were localized to chromosomes $9,15,18$, and 21 , respectively.

Of the 4 chromosomes identified to contain FGF-7 sequences, we were able to localize the gene that encodes FGF-7 to chromosome 15. The structure of the gene was deduced from the $18,451.822$ kbp chromosome 15 reference genomic contig (NCBI Accession NT_010194.13). The fibroblast growth factor 7 gene is characterized by a length of at least $64.065 \mathrm{kbp}$, a presence on both chromatids of chromosome 15 , a cytogenetic localization of 15q15-q21.1 at 41.2 +/- 13.3 MB, and 
4 exons that generate a 3,852 $\mathrm{kb}$ mRNA. The arrangement of introns and exons is displayed in Table 2. Exons 2 and 3 are separated by an unusually large intron (58.567 kbp) that also splits the codon for Asn67 (underlined and bold type in Table 2). Fifty-seven single nucleotide polymorphisms (SNP) for this gene are present in NCBI's public SNP database (Human Genome Build 31, February 28, 2003).

The extent to which introns 2 and 3, and exons 3 and 4, of the chromosome 15 FGF-7 were distributed among chromosomes 9, 18, and 21 was also investigated (Table 3 ). The size of exons 3 and 4 were each almost perfectly conserved among chromosomes 18,21 , one region located in the middle of the $\mathrm{p}$ arm $(\mathrm{Chr} 9 \mathrm{pm})$, and two regions proximal to the centromere $(\mathrm{Chr} 9 \mathrm{qc})$ and telomere (Chr 9qt) of the $\mathrm{q}$ arm of chromosome 9. The length of the chromosome 18 FGF-7 sequence is nearly identical to the length of the chromosome 15 FGF-7 gene sequence. Scattered over these 3 chromosomes are 7 regions that exhibit near-identical sequences. We considered these regions to be duplications of the FGF-7 gene sequences present on chromosome 15. These duplicated copies contain only portions of the FGF-7 gene sequence (Figure 1). Such duplications for the FGF-7 gene have been previously detected by Southern blotting (38) and by fluorescence in situ hybridization (39). In this latter study, the duplications were visualized on chromosomes 2, 9 (two spots), 15, 18 (two spots), and 21 (two spots). Our sequence analysis differs from these results in that no sequences were identified to account for the chromosome 2 spot.

Five copies of the duplicated sequences were localized to chromosome 9 (Table 3 and Figure 1). Among the conserved copies, two gap-containing sequences were identified proximal to the centromere on the 9p arm (9pc) and proximal to the telomere on the $9 q$ arm $(9 p t)$. 


\section{Analysis of Human Urinary Bladder FGF-7 Amino Acid Sequence}

Figure 2 displays the deduced amino acid sequence for the 173 aa recombinant rFGF7-His protein. The $\mathrm{N}$-terminus was engineered to contain a Met residue to provide a codon for initiation of translation in E. coli. The C-terminus was engineered to contain a His-hexamer to provide for detection during biological assays and for isolation of the recombinant protein by metal-chelate affinity chromatography. The calculated $\mathrm{M}_{\mathrm{r}}$ and isoelectric point of rFGF7-His is 20,151 and 9.58, respectively.

Motifs and sites for post-translational modification that are predicted to occur in the wild-type FGF-7 sequence are also shown in Figure 2. Naturally occurring FGF-7 proteins are predicted to contain sites for N-linked glycosylation (Asn16), casein kinase II phosphorylation site (Ser18 and Thr150), phosphorylation site by protein kinase C (Ser124) and amidation (Gly147). A 24 aa region (Gly96-Tyr119) is the consensus sequence for the FGF family of heparin-binding polypeptides (40) and a potential transmembrane segment (41). The "glycine box" sequence (NQKGIPVRG, residues 139 - 147) (42) is the major determinant for the specificity of the binding of FGF-7 to heparan sulfate-FGFR complexes (40). Residues that have been implicated in binding to heparin (23) (1) were identified as Arg43, Asn117, Asn139, Gln140, Val145,Lys148, Asn154, Lys155, and Thr156 (Figure 2). These residues form a positively charged motif that is present on the surface of the macromolecule (Supplemental Figure 2). The heparin-binding motif of rFGF7-His is therefore exposed to the solvent, and, more importantly, available to bind to and interact with heparan-, chondroitin-, or dermatin- sulfate proteoglycans. 


\section{Expression, Isolation, and Characterization of Recombinant FGF-7 in Escherichia coli}

The expression of recombinant FGF-7 was realized as a fusion protein with a hexamer of His residues at the C-terminus (Figure 2 and Supplemental Figure 2). This recombinant fusion protein, designated rFGF7-His, was found to comprise $1-2 \%$ of the total cellular protein and to partition equally between the soluble and insoluble fractions of lysed BL21trxB(DE3) E. coli (data not shown). We pursued the characterization of the soluble form of rFGF7-His with respect to 1) intactness, 2) ability to interact with heparin, 3) folding, and 4) biological activity in vitro.

rFGF7-His was determined to be full-length because a) amino acid sequencing of the Nterminus (ACNDMTPEQMATNV) of the isolated protein was found to be consistent with the Nterminus of the mature form of the NCBI reference sequence NM_002009.2 and b) the C-terminal His hexamer conferred the ability of rFGF7-His to bind avidly to nickel-chelate affinity resins (Figure 3, lane 1).

rFGF7-His was judged to be folded properly because a) it partitioned into the soluble phase of the bacterial extract, b) the isolated, non-reduced form exhibited a greater mobility on SDS-PAGE than the isolated, reduced form (data not shown) and c) it bound to heparin (Figure 3, lane 2). Soluble rFGF7-His was isolated as a monomeric polypeptide of $\sim 24 \mathrm{kDa}$ by nickel-chelate (Figure 3, lane 1) and heparin affinity chromatographies (Figure 3, lane 2). The ability of soluble rFGF7His to interact with nickel-chelate resins through its C-terminal His-hexamer (Figure 3, lane 1) demonstrates that this His- hexamer is localized to the periphery of the folded protein, in agreement with the crystal structure of the human (23) and rat (1) FGF-7 proteins. Concerns that manipulation of the C-terminal region of rFGF7-His would influence its folding and biological activity (43) prompted us to ask if our preparations of rFGF7-His would bind to heparin-affinity resins in the same manner as reported in the literature. Figure 3 (lane 2) demonstrates that rFGF7-His elutes from heparin-affinity chromatography columns at $1.0 \mathrm{M} \mathrm{LiCl}$, an ionic strength in agreement with 
that reported for native (21) or rFGF-7 (44). Sequential chromatography on heparin-affinity followed by nickel-chelate affinity chromatographies (Figure 3, lane 3), or the converse (nickelchelate followed by heparin, Figure 3, lane 4) clearly demonstrate that the presence of the Cterminal His-hexamer does not impact the ability of the recombinant protein to interact with heparin or fold into a conformationally stable state. Stabilization of rFGF7-His was found to be enhanced by inclusion of heparin in the storage buffer solution (not shown).

\section{Biological Activities of Recombinant FGF-7}

rFGF7-His was found to exhibit mitogenic activity on human urothelial cells in vitro (Figure 4). Cultures of urothelial cells that attained confluence by incubation in growth media reduced the rate of $\left[{ }^{3} \mathrm{H}\right]$-thymidine incorporation from $37,600 \pm 900 \mathrm{cpm}$ to $6,000 \pm 360 \mathrm{cpm}$ after switching to starvation medium (Figure 4B). Addition of increasing concentrations of rFGF7-His to cells in starvation medium resulted in a dose-dependent increase of incorporation of $\left[{ }^{3} \mathrm{H}\right]$-thymidine into urothelial cell DNA. A 5.6-fold increase in incorporation was observed at a concentration of $0.1 \mathrm{ng}$ $\mathrm{ml}^{-1}$, relative to no input growth factor. The mitogenic activity of rFGF7-His was dependent on the absence of input heparin. This activity was observed only with confluent cultures, whereas nonconfluent cultures resulted in non-significant levels of incorporation (data not shown). The culture in Figure 4A was confluent and displayed the typical cobblestone appearance of urothelial cells in vitro, as compared to published photographs (8). Atop Figure 4A is a "giant" cell with two prominent nuclei - this urothelial cell-subtype corresponds to the large, polygonal suprabasal cell that comprise the lumenal layer of transitional epithelium. Suprabasal urothelial cells are the largest epithelial cells in mammals. Secretory granules, lipid inclusions, and lysosomes are prominent in these suprabasal cells, which are also positive for uroplakin (data not shown). 
rFGF-7 (Palifermin) was also found to exhibit mitogenic activity on the urothelial cells of murine transitional epithelium (Figure 5). For these experiments, $100 \mu \mathrm{g}$ of rFGF-7 (Palifermin) in vehicle was injected each day for 14 days into the neck folds of C57BL/6J mice. Subsequent histological staining with Mason's trichrome (data not shown) revealed that animals treated with rFGF-7 (Palifermin) underwent a dramatic expansion of the number of urothelial cell layers of urinary bladder transitional epithelium (data not shown). A layer of stratified urothelium 15-25 cells thick characterized this expansion. In contrast, control mice that received vehicle only did not exhibit detectable urothelial expansion, but instead displayed a normal layer of 2-5 cells thick (data not shown).

Immunostaining with antibodies specific for the nuclear protein Ki-67, an established marker of epithelial cell proliferation $(45,46)$, demonstrated that basal urothelial cells were traversing the cell cycle (Figure 5). The Ki-67 signal persisted throughout the period of rFGF-7 (Palifermin) administration and was prevalent in all animals tested $(\mathrm{n}=3)$. A Ki-67 signal was observed to decrease in intensity as cells detached from the urothelial basement membrane and migrated towards the bladder lumen. Animals receiving vehicle did not exhibit Ki-67 immunoreactivity. The positive correlation between rFGF-7 (Palifermin) administration, expansion of the layers of transitional epithelium, and Ki-67 immunoreactivity confirms that FGF-7 is a positive regulator of urothelial cell proliferation.

\section{FGF-7 is a Non-Essential Mitogen During Partial Bladder Outlet Obstruction}

An experimental mouse model of partial bladder outlet obstruction was developed wherein a partial surgical ligature was placed around the urinary bladder outlet. In this model, animals that underwent partial ligation exhibited bladders that were abnormally distended. The hallmark feature of transitional epithelium over the 8 days of obstruction was the marked increase of stratification of 
transitional epithelium and of urothelial cells traversing the cell cycle. The mitogenic signal that stimulated the exit of these cells from the $G_{O}$ phase and the progression through the cell cycle was hypothesized to originate in the mesenchyme, and that this principal mesenchymal factor was FGF7.

To test our hypothesis, a comparison of the urothelial response to obstruction between wild-type and FGF-7 null mice was performed. It was assumed that this response would involve individual urothelial cells undergoing proliferation. Accordingly, urothelial cells traversing the cell cycle were detected by immunostaining with polyclonal antibodies specific to the Ki-67 nuclear antigen, an accepted marker of proliferating epithelial cells.

Quantification of total and proliferating urothelial cells was achieved by analysis using the principles of unbiased stereology. If mesenchymal FGF-7, produced and secreted by fibroblasts of the lamina propria, was the principal mitogen that stimulated the proliferation of urothelial cells, then lack of this major paracrine factor in FGF7-null mice should influence the urothelial response to injury by exhibiting a measurable decrease in the percentage of proliferating urothelial cells, as compared to wild-type mice.

The following transitional epithelia exhibited 2-5 layers of urothelial cells that typify the normal phenotype and were non-reactive with antibodies specific for the Ki-67 antigen: wild-type normal, FGF7-null normal, wild-type sham, and FGF7-null sham (data not shown). These results indicate that urothelial cells were not removed from the $G_{0}$ resting phase of the cell cycle by surgical procedures that did not include urethral ligation.

Mice that underwent ligation for 1-2 days exhibited minimally detectable urothelial expansion (data not shown). Ligation for 4 days elicited subtle, but detectable, urothelial expansion (data not shown). The most consistent and reproducible efforts required 8 days of partial obstruction. Partially obstructed bladders after 8-days post-ligation were prominently larger and distended than 
control animals (data not shown), a result that indicated that partial outlet obstruction of the bladder was successfully accomplished by ligation of the urethra.

In contrast to control and sham-operated animals, transitional epithelia from animals that underwent partial urethral ligation at the bladder neck exhibited marked immunoreactivity with antibodies specific for the Ki-67 nuclear antigen. The total number of Ki-67 positive cells, as well as the total number of urothelial cells, was determined by stereology (Figure 6). Calculation of mean values is presented in Table 4. The percentage of Ki-67 positive cells for obstructed wild-type and FGF7-null mice was calculated to be $1.78 \pm 0.46$ cells and $1.60 \pm 0.94$, respectively. A statistical comparison between these two groups with the student's t-test indicated that this difference was not significant.

\section{FGF-10 is a Compensatory Mitogen During Partial Bladder Outlet Obstruction}

Bladder specimens from mice subjected to partial outlet obstruction as described above were tested for FGF-10 expression. FGF7- null and WT mice were compared for differential FGF-10 expression among fibroblasts within the lamina propria using the same stereologic analysis as described above. The mean number of FGF-10 positively stained fibroblasts for the WT and the FGF7-null mice per bladder were 195 and 1086, respectively (Table 4). This difference was statistically significant $(p=0.004)$ proving that FGF-10 expression was up-regulated in response to obstruction. The collective data support the hypothesis that FGF-10 is an essential and compensatory mitogen for murine urothelium turnover.

\section{DISCUSSION}

In animal models, partial outlet obstruction of the urinary bladder induced by urethral ligation elicits a 3-phase programmed response: (i) an initial hypertrophy phase, (ii) a compensatory phase, and 
(iii) a decompensatory phase $(18,47)$. The early molecular response of the lamina propria and urothelium in response to outlet obstruction has been studied in the context of mesenchymalepithelial interactions, specifically the role of mesenchymal growth factors in the induction of urothelial cell proliferation $(13,15,48-50)$. In these models, FGF-7 mRNA has consistently been observed to be markedly upregulated in response to obstruction $(14,15,47)$. Our analysis of the FGF-7 gene indicated that while exons 3 and 4 were duplicated within the human genome, we found no evidence that full-length FGF-7 mRNAs could arise from any other locus than 15q15$\mathrm{q} 21.1$.

Mesenchymal mediators of epithelial cell behavior, proliferation, and differentiation represent an important means of regulating development, homoeostasis, and the response to injury. In the urinary bladder, the paracrine mediator that has been most studied is FGF-7, a polypeptide synthesized and secreted by fibroblasts of the lamina propria $(15,20,47-50)$. Our study demonstrates that this growth factor is a mitogen for human and murine urothelial cells of transitional epithelium, a result in agreement with prior reports that studied the effect of the purified factor on transitional epithelia from monkey $(51)$, rat $(51,52)$, and murine $(20,35)$ sources.

Equivalent biological activities were observed for rFGF7-His (our preparations) and rFGF-7 (provided by Amgen as the pharmaceutical Palifermin) in assays that monitored the incorporation of $\left[{ }^{3} \mathrm{H}\right]$-thymidine into the DNA of human urothelial cells in vitro. The mitogenic activity of rFGF7His on human urothelial cells was observed only with confluent cultures, a phenomenon previously reported for normal human keratinocytes (53). Our data is also in agreement with prior reports (44, 54) that demonstrated that heparin was inhibitory to the stimulatory activity of rFGF-7 on Balb/MK keratinocytes.

Since the initial response of the bladder to outlet obstruction is to turnover its urothelium via reciprocal mesenchymal-epithelial interactions $(14,15,47)$, we asked to what extent 
mesenchymally-derived FGF-7 regulates urothelial cell proliferation in response to obstruction. To answer this question, we quantified the proliferative response to obstruction by stereological analysis of Ki-67 staining of the urothelium. Not only were the total number of Ki-67 positive cells nearly equivalent between the wild-type and FGF7-null sets of mice, but the percentage of urothelial cells traversing the cell cycle between these two sets was statistically insignificant. We noted that the urothelial response to obstruction was variable from animal to animal, an observation shared by other laboratories $(15,16,55-57)$ and noted clinically as well.

The collective data prove our hypothesis that redundant mechanisms compensate for the lack of FGF-7 in FGF7-null mice that continue to exhibit proliferating urothelial cells in response to obstruction. The principal mitogenic candidate to compensate for the absence of FGF-7 was shown to be FGF-10, a known mitogen for urothelial cells in vitro and in vivo $(13,25)$. Since both FGF-7 and FGF-10 can bind to, interact with, and stimulate a mitogenic signaling pathway through the same FGFR2 isoform 2 receptor (58), we conclude that FGFR2 isoform 2 remains functional in FGF7-null mice. 
Our prior report that the FGFR2 isoform 2 receptor is expressed by the superficial layer of transitional epithelia (25) is clinically significant because intravesical instillation of recombinant FGF-7 and -10 into the bladder lumen is predicted to trigger urothelial repair in response to injury due to obstruction. In addition, clinical conditions involving the urinary tract which cause epithelial damage such as infections and trauma leading to stricture disease, erosive disorders such as interstitial cystitis, and urinary tract disruption from transurethral resection surgery could be treated with mitogenic stimulation. Ongoing work is expected to lead to a better understanding of the steady-state interrelationships that involve urothelial cells, growth factors, matricellular proteins, the extracellular matrix, and the urothelial basement membrane. Advancing the study of FGF-7 and -10 is relevant because of the polypeptides' potential use as a clinical tool to treat, and ultimately cure, a variety of lower urinary tract conditions and diseases.

\section{MATERIALS AND METHODS}

\section{Materials}

Human urinary bladder cDNA was obtained from Invitrogen (Carlsbad, CA). Oligonucleotide primers were synthesized by Keystone Laboratories (Foster City, CA). Escherichia coli strains NovaBlue and BL21trxB(DE3), Perfectly Blunt Cloning Kit, plasmid pET21d, Bug Buster lysis reagent, and carbenicillin were purchased from Novagen (Madison, WI). Restriction enzymes NcoI and XhoI, T4 DNA ligase, Taq polymerase, and Complete Protease Inhibitors were from Roche Molecular Biochemicals (Indianapolis, IN). Ni-NTA metal-chelate affinity resin was from Qiagen (Chatsworth, CA). HiTrap heparin-sepharose affinity resins were from Amersham Biochemicals (Piscataway, NJ). SDS-PAGE gels and the Amplified Alkaline Phosphatase ImmunoBlot Assay detection system were obtained from BioRad (Hercules, CA). Tetracycline and kanamycin were from Calbiochem (La Jolla, CA). Recombinant FGF-7 (rFGF-7, Palifermin, rHuKGF)) was provided by Amgen (Thousand Oaks, CA). Rabbit anti-bovine uroplakin immunoglobulins were 
obtained from Dr. H. Sun (New York University, New York, NY). Rabbit anti-Ki67

immunoglobulins were obtained from Novocastra (Newcastle, UK). Mouse anti-FGF10

immunoglobulins were purchased from R\&D Systems (Minneapolis, MN). Goat anti-mouse

horseradish-peroxidase conjugated immunoglobulins were obtained from Jackson ImmunoResearch Laboratories (West Grove, PA).

\section{Isolation of rFGF7-His}

Two types of recombinant (r) FGF-7 were used in this study. rFGF7-His, prepared in our laboratory as described in the Supplemental Materials Section and Palifermin (rFGF-7 lacking a C-terminal His-tag), prepared and provided to us by Amgen (59).

\section{N-terminal Amino Acid Sequencing of rFGF7-His}

A $1 \mathrm{ml}$ fraction eluted from a nickel-chelate affinity chromatography column that contained 25 $\mu \mathrm{g} \mathrm{ml} \mathrm{m}^{-1}$ of $\mathrm{rFGF} 7-H i s$ was precipitated with trichloroacetic acid, washed, dried and dissolved in $0.125 \mathrm{M}$ Tris- $\mathrm{HCl}$ (pH 6.8), 2\% (w/v) SDS, 10\% glycerol, $0.05 \%$ bromphenol blue, $0.05 \mathrm{M}$ dithiothreitol. After heating to $37^{\circ} \mathrm{C}$ for 20 minutes, the fraction was electrophoresed through a $15 \%$ polyacrylamide gel that contained $0.1 \%$ SDS. Thioglycolate $(0.1 \mathrm{mM})$ was present in the upper buffer chamber to scavenge unpolymerized acrylamide that could potentially result in blockage of the N-terminus. Following electrophoresis, fractionated proteins were electrotransferred to a polyvinylidene difluoride membrane in 0.01 M MOPS ( $\mathrm{pH} 11.0$ ) that contained 20\% methanol, visualized by staining with Coommasie Brilliant Blue R-250 and destained. The rFGF7-His band was excised from the membrane, and sequenced by Edman degradation with an Applied Biosystems 477A protein sequencer. 


\section{[3H-Thymidine Incorporation Assays of Urothelial Cell Proliferation}

Because of the use of human transitional epithelial tissue for this study, these experiments were reviewed by the Institutional Review Board of Children's Hospital and Regional Medical Center.

Primary cultures of bladder urothelial cells derived from surgical explants were grown as previously described (13). Assays of DNA synthesis of bladder urothelial cells were performed as

previously described (13). Incorporation of $\left[{ }^{3} \mathrm{H}\right]$-thymidine into urothelial cell DNA as a function of rFGF7-His was inhibited by $10 \mu \mathrm{g} \mathrm{ml}^{-1}$ heparin, in agreement with a prior report $(43,54)$, presumably because of the influence of extracellular matrix components present in the cultures.

Our preparations of rFGF7-His from soluble extracts were biologically active and the engineering of a C-terminal hexamer of histidine residues did not affect solubility, folding, or ability to bind to heparin. However, we found that the yields of bacterial rFGF7-His were disappointing, a result in agreement with a prior report that established that the growth factor exhibited limited stability in aqueous media, as it undergoes denaturation followed by aggregation at $37{ }^{\circ} \mathrm{C}(60)$. Therefore, we used Amgen's preparations of active recombinant FGF-7 for use in assays where milligram quantities of the growth factor were needed, e.g. injection into mice.

\section{Induction of Murine Urothelial Cell Proliferation}

rFGF-7, lacking a C-terminal histidine tag and ending at $\mathrm{Thr}_{165}$ (Figure 4), was obtained from Amgen as the pharmaceutical Palifermin, and dissolved in vehicle $(120 \mathrm{mM} \mathrm{NaCl}, 2.7 \mathrm{mM} \mathrm{KCl}, 4$ $\mathrm{mM} \mathrm{NaH}{ }_{2} \mathrm{PO}_{4}(\mathrm{pH} 7.4), 5 \mu \mathrm{g} \mathrm{ml}^{-1}$ heparin) at a final growth factor concentration of $100 \mu \mathrm{g} \mathrm{ml}^{-1}$. One $\mathrm{ml}$ was administered daily by intraperitoneal injection into the neck folds of wild-type C57BL/6J mice. On day 15, mice were euthanized and their bladders were harvested en bloc by anatomical positioning on a paraffin block. Specimens were fixed with methyl Carnoy's solution for 
16-24 hr, transferred to $120 \mathrm{mM} \mathrm{NaCl}, 2.7 \mathrm{mM} \mathrm{KCl}, 4 \mathrm{mM} \mathrm{NaH}_{2} \mathrm{PO}_{4}(\mathrm{pH} 7.4$ ), embedded in paraffin, cut into 5-10 $\mu \mathrm{m}$ thick sections, and processed by Ki-67 immunohistochemistry (20) or visualized with Masson's trichrome stain.

\section{Mouse Model of Bladder Outlet Obstruction}

The Institutional Animal Care and Use Committee of Children's Hospital and Regional Medical Center approved these experiments. Wild-type C57BL/6J mice were obtained from B and K (Kent, WA). FGF7-null mice (33) of the same parental inbred strain were provided by Dr. E. Fuchs (The Rockefeller University). All mice were 8-week old females.

Two sets of mice were used: wild-type and FGF7-null. Each set consisted of 3 groups: (1) a control group ( $\mathrm{n}=2)$ that did not undergo any type of surgery; (2) a second control group $(\mathrm{n}=2)$ that underwent a sham operation, i.e. abdominal cavity was opened, a 24 gauge angiocatheter was inserted into the bladder via the urethra and then removed, and the cavity was closed; and (3) a group $(\mathrm{n}=8)$ that underwent ligation at the bladder neck area. The partial ligation was performed with mice under anesthesia. Once adequate anesthesia was obtained, the abdominal cavity was opened, exposing the bladder and urethra. A 26-gauge angiocatheter was inserted into the bladder via the urethra and a 7-0-monofilament polyglyconate suture was tied around the urethra at the bladder neck. The angiocatheter was then removed and reinserted to ensure partial outlet obstruction was obtained. Partial outlet obstruction was confirmed by observing urine flow with gentle extrinsic bladder compression. The abdomen was then closed, mice were allowed to recover, and housed for 8 additional days. Mice were sacrificed; the bladder was excised, pinned to a paraffin block, fixed in Methyl Carnoy's solution, and sectioned according the principles of stereology (below). 


\section{Immunostaining with antibodies specific for Ki-67 and FGF-10}

Specimens were fixed with methyl Carnoy's solution for 16-24 hr, transferred to $120 \mathrm{mM} \mathrm{NaCl}, 2.7$ $\mathrm{mM} \mathrm{KCl}, 4 \mathrm{mM} \mathrm{NaH}{ }_{2} \mathrm{PO}_{4}(\mathrm{pH} 7.4)$, embedded in paraffin, cut into 5-10 $\mu \mathrm{m}$ thick sections, and processed by Ki-67 immunohistochemistry (20) or visualized with Masson's trichrome stain. Diaminobenzidine colorimetric staining with primary monoclonal mouse anti-human FGF-10 IgG at a concentration of $15 \mu \mathrm{g} \mathrm{ml}^{-1}$ and goat anti-mouse horseradish-peroxidase conjugated $\mathrm{IgG}$ at 1:500 dilution was performed with 5\% goat serum block. Slides were counter-stained with a 1:10 dilution of Gill's formula for 1 minute and fixed.

\section{Stereology Procedures}

Estimates of the number of total and proliferating (Ki-67 positive) urothelial cells, and FGF-10 positive fibroblast cells, of obstructed murine bladders were determined by use of the Optical Fractionator (61). The Optical Fractionator method combines a 3-dimensional unbiased counting frame (the optical disector) and an unbiased sampling method (the fractionator) to generate estimates of the total number of particles of interest, in this case Ki-67 positive urothelial cells and FGF-10 positive fibroblasts. The ensuing estimate is unbiased and free of volume artifacts commonly present with other counting methods (62). Implementation of the Optical Fractionator was performed using a BH-2 Olympus microscope equipped with an Optronics DEI 750 digital video camera and Ludl high precision motorized stage. Data collection and microscope operation was controlled by Stereo Investigator software (version 3.19, MicroBrightField, Williston, Vermont).

The application of the fractionator in the present sampling design consisted of initially systematically collecting every $40^{\text {th }}$ section across the entire bladder starting with a random start (e.g. a random start might be 12 , therefore collect the $12,52,92 \ldots$ sections). Hence this set of 
sections (and the structure of interest contained within, i.e., the obstructed bladder) represented $1 / 40^{\text {th }}$ of the entire structure. Following staining of this set of sections, Ki-67 positive urothelial cells were counted in a known aerial fraction of the transitional epithelial layer. The aerial fraction was determined by placing a random stepping grid, generated by the Stereo Investigator software program, over the section. An appropriate stepping grid (500 x $500 \mu \mathrm{m}$, or an area of $\left.250,000 \mu \mathrm{m}^{2}\right)$ was used to place sequential disectors across the surface of the sections. A disector frame $(10 \times 10=$ $100 \mu \mathrm{m}^{2}$ ) was placed in each stepping grid and used to count Ki-67 positive cells according to optical disector counting rules. Immunopositive cells were readily identified by the dark brown coloration of the nuclei characteristic of the staining procedures (see Figure 5 in manuscript). The overlay of this stepping grid was placed randomly (independently) over the transitional epithelium, thus ensuring that each step was systematic and random. In our experiments, the optical disectors sampled $1 / 2500^{\text {th }}$ of the transitional epithelium. Combined with the $1 / 40^{\text {th }}$ of the structure represented by the section, the optical disectors sampled $1 / 100,000^{\text {th }}$ of the entire structure. Cell counts were summed from all disectors across all sections collected, and the total number was determined by multiplying the number counted by the inverse of the fraction sampled. Systematic random sampling has been determined to be fair because every part of the original structure had an equal probability of being selected due to the random position set within the first interval, and systematic random sampling offers additional advantages of efficiency (63).

FGF-10 positive cells were counted that were clearly labeled, possessed the characteristic shape of a fibroblast cell, and did not intercept the top of the tissue section in what was judged to be the widest cell profile. Statistical differences between FGF-7 knock-out mice and wild type mice were determined using Student's t-test, SPSS base 10 (Mountain View, CA) statistical software. Tests for homogeneity of group variance and normal distributions indicated that the non-parametric 
Mann-Whitney test for two-group means comparison was the appropriate statistical test for FGF-10 expression analysis.

\section{ACKNOWLEDGEMENTS}

This work was supported by the Division of Pediatric Urology, Seattle Children's Hospital Foundation, and NIH grant 1R01-DK62251-05 (J.A.B.). Palifermin (recombinant human FGF-7, recombinant human keratinocyte growth factor) was a generous gift from Amgen (Thousand Oaks, CA).

\section{SUPPLEMENTAL INFORMATION}

Supplementary information is available online at The Journal of Clinical Investigation website.

\section{REFERENCES}

1. Ye, S., Luo, Y., Lu, W., Jones, R.B., Linhardt, R.J., Capila, I., Toida, T., Kan, M., Pelletier, H., and McKeehan, W.L. 2001. Structural basis for interaction of FGF-1, FGF-2, and FGF7 with different heparan sulfate motifs. Biochemistry 40:14429-14439.

2. Wein, A.J. 1995. Bladder outlet obstruction--an overview. Adv.Exp.Med.Biol. 385:3-5.

3. Blaivas, J.G. 1996. The bladder is an unreliable witness. Neurourol.Urodyn. 15:443-445.

4. Hainau, B. and Dombernowsky, P. 1974. Histology and cell proliferation in human bladder tumors. An autoradiographic study. Cancer 33:115-126.

5. Hicks, R.M. 1975. The mammalian urinary bladder: an accommodating organ.

Biol.Rev.Camb.Philos.Soc. 50:215-246.

6. Lindner, P., Bauer, K., Nieba, L., Kremmer, E., Krebber, C., Honegger, A., Klinger, B., Mocikat, R., and Plueckthun, A. 1997. Specific detection of his-tagged proteins with recombinant anti-his-tag scFv-phosphatase or scFv-phage fusions. Biotechniques 22:140149.

7. Miki, T., Fleming, T.P., Bottaro, D.P., Rubin, J.S., Ron, D., and Aaronson, S.A. 1991. Expression cDNA cloning of the KGF receptor by creation of a transforming autocrine loop. Science 251:72-75. 
8. Southgate, J., Hutton, K.A., Thomas, D.F., and Trejdosiewicz, L.K. 1994. Normal human urothelial cells in vitro: proliferation and induction of stratification. Lab.Invest. 71:583-594.

9. Freeman, M.R., Yoo, J.J., Raab, G., Soker, S., Adam, R.M., Schneck, F.X., Renshaw, A.A., Klagsbrun, M., and Atala, A. 1997. Heparin-binding EGF-like growth factor is an autocrine growth factor for human urothelial cells and is synthesized by epithelial and smooth muscle cells in the human bladder. J.Clin.Invest. 99:1028-1036.

10. Teng, J., Wang, Z.Y., and Bjorling, D. 2001. Stimulation of human urothelial cell proliferation by estrogen receptor activation. Urology 57:102-103.

11. Teng, J., Wang, Z.Y., and Bjorling, D.E. 2002. Estrogen-induced proliferation of urothelial cells is modulated by nerve growth factor. Am.J.Physiol Renal Physiol 282:F1075-F1083.

12. De Boer, W.I., Vermeij, M., Diez, d.M.S., Bindels, E., Radvanyi, F., van der Kwast, T., and Chopin, D. 1996. Functions of fibroblast and transforming growth factors in primary organoid-like cultures of normal human urothelium. Lab.Invest. 75:147-156.

13. Bagai, S., Rubio, E., Cheng, J.F., Sweet, R., Thomas, R., Fuchs, E., Grady, R., Mitchell, M., and Bassuk, J.A. 2002. Fibroblast growth factor-10 is a mitogen for urothelial cells. J.Biol.Chem. 277:23828-23837.

14. Bassuk, J.A., Grady, R., and Mitchell, M. 2000. Review article: The molecular era of bladder research. Transgenic mice as experimental tools in the study of outlet obstruction. $J$ Urol 164:170-179.

15. Baskin, L.S., Sutherland, R.S., Thomson, A.A., Hayward, S.W., and Cunha, G.R. 1996. Growth factors and receptors in bladder development and obstruction. Lab Invest 75:157166.

16. Mattiasson, A. and Uvelius, B. 1982. Changes in contractile properties in hypertrophic rat urinary bladder. J.Urol. 128:1340-1342.

17. Monson, F.C., McKenna, B.A., Wein, A.J., and Levin, R.M. 1992. Effect of outlet obstruction on 3H-thymidine uptake: a biochemical and radioautographic study. J.Urol. 148:158-162.

18. Levin, R.M., Memberg, W., Ruggieri, M.R., and Wein, A.J. 1986. Functional effects of in vitro obstruction on the rabbit urinary bladder. J.Urol. 135:847-851.

19. Levin, R.M., Haugaard, N., Mogavero, L., Leggett, R.E., and Das, A.K. 1999. Biochemical evaluation of obstructive bladder dysfunction in men secondary to BPH: a preliminary report. Urology 53:446-450.

20. Bassuk, J.A., Cochrane, K., and Mitchell, M.E. 2003. Induction of urothelial cell proliferation by fibroblast growth factor-7 in RAG1-deficient mice. Adv.Exp Med.Biol. 539:623-633. 
21. Rubin, J.S., Osada, H., Finch, P.W., Taylor, W.G., Rudikoff, S., and Aaronson, S.A. 1989. Purification and characterization of a newly identified growth factor specific for epithelial cells. Proc.Natl.Acad.Sci.U.S.A. 86:802-806.

22. Rubin, J.S., Bottaro, D.P., Chedid, M., Miki, T., Ron, D., Cheon, G., Taylor, W.G., Fortney, E., Sakata, H., and Finch, P.W. 1995. Keratinocyte growth factor. Cell Biol Int 19:399-411.

23. Osslund, T.D., Syed, R., Singer, E., Hsu, E.W., Nybo, R., Chen, B.L., Harvey, T., Arakawa, T., Narhi, L.O., Chirino, A. et al . 1998. Correlation between the 1.6 A crystal structure and mutational analysis of keratinocyte growth factor. Protein Sci. 7:1681-1690.

24. Olsen, S.K., Garbi, M., Zampieri, N., Eliseenkova, A.V., Ornitz, D.M., Goldfarb, M., and Mohammadi, M. 2003. FHFs share structural but not functional homology to FGFs. J.Biol.Chem.

25. Zhang, D., Kosman, J., Jacobs, N., Grady, R., and Bassuk, J. 2006. Fibroblast growth factor-10 and the isoform 2 receptor of the FGFR2 gene exhibit bi-directional paracrine targeting and co-translocation into urothelial cell nuclei in normal and exstrophic human urinary bladder. Am.J.Pathol. 291:F481-F494.

26. Jang, J.H., Wang, F., and Kan, M. 1997. Heparan sulfate is required for interaction and activation of the epithelial cell fibroblast growth factor receptor-2IIIb with stromal- derived fibroblast growth factor-7. In Vitro Cell Dev.Biol.Anim. 33:819-824.

27. McKeehan, W.L., Wang, F., and Kan, M. 1998. The heparan sulfate-fibroblast growth factor family: Diversity of structure and function. Prog.Nucl.Acid Res.Mol.Biol. 59:135176.

28. Trowbridge, J.M., Rudisill, J.A., Ron, D., and Gallo, R.L. 2002. Dermatan sulfate binds and potentiates activity of keratinocyte growth factor (FGF-7). J.Biol.Chem. 277:42815-42820.

29. Sannes, P.L., Khosla, J., Li, C.M., and Pagan, I. 1998. Sulfation of extracellular matrices modifies growth factor effects on type II cells on laminin substrata. Am.J.Physiol 275:L701L708.

30. Miki, T., Bottaro, D.P., Fleming, T.P., Smith, C.L., Burgess, W.H., Chan, A.M., and Aaronson, S.A. 1992. Determination of ligand-binding specificity by alternative splicing: two distinct growth factor receptors encoded by a single gene. Proc.Natl.Acad.Sci.U.S.A. 89:246-250.

31. Spivak-Kroizman, T., Lemmon, M.A., Dikic, I., Ladbury, J.E., Pinchasi, D., Huang, J., Jaye, M., Crumley, G., Schlessinger, J., and Lax, I. 1994. Heparin-induced oligomerization of FGF molecules is responsible for FGF receptor dimerization, activation, and cell proliferation. Cell 79:1015-1024.

32. Oldridge, M., Zackai, E.H., McDonald-McGinn, D.M., Iseki, S., Morriss-Kay, G.M., Twigg, S.R., Johnson, D., Wall, S.A., Jiang, W., Theda, C. et al . 1999. De novo alu-element insertions in FGFR2 identify a distinct pathological basis for Apert syndrome. Am.J.Hum.Genet. 64:446-461. 
33. Guo, L., Degenstein, L., and Fuchs, E. 1996. Keratinocyte growth factor is required for hair development but not for wound healing. Genes Dev. 10:165-175.

34. Qiao, J., Uzzo, R., Obara-Ishihara, T., Degenstein, L., Fuchs, E., and Herzlinger, D. 1999. FGF-7 modulates ureteric bud growth and nephron number in the developing kidney. Dev.Suppl. 126:547-554.

35. Tash, J.A., David, S.G., Vaughan, E.D.E., and Herzlinger, D.A. 2001. Fibroblast growth factor-7 regulates stratification of the bladder urothelium. J Urol JID - 0376374 166:25362541 .

36. Min, H., Danilenko, D.M., Scully, S.A., Bolon, B., Ring, B.D., Tarpley, J.E., DeRose, M., and Simonet, W.S. 1998. Fgf-10 is required for both limb and lung development and exhibits striking functional similarity to Drosophila branchless. Genes Dev. 12:3156-3161.

37. Bassuk,J.A. 2006. Proteins as Signals in Urothelial Cell Proliferation and the Response to Injury. American Urological Association / Society of Basic Urological Research Summer Research Conference August 3-5, 2006: (Abstr.)

38. Kelley, M.J., Pech, M., Seuanez, H.N., Rubin, J.S., O'Brien, S.J., and Aaronson, S.A. 1992. Emergence of the keratinocyte growth factor multigene family during the great ape radiation. Proc.Natl.Acad.Sci.U.S.A. 89:9287-9291.

39. Zimonjic, D.B., Kelley, M.J., Rubin, J.S., Aaronson, S.A., and Popescu, N.C. 1997. Fluorescence in situ hybridization analysis of keratinocyte growth factor gene amplification and dispersion in evolution of great apes and humans. Proc Natl Acad Sci U S A JID 7505876 94:11461-11465.

40. Luo, Y., Lu, W., Mohamedali, K.A., Jang, J.H., Jones, R.B., Gabriel, J.L., Kan, M., and McKeehan, W.L. 1998. The glycine box: a determinant of specificity for fibroblast growth factor. Biochemistry 37:16506-16515.

41. Persson, B. and Argos, P. 1994. Prediction of transmembrane segments in proteins utilising multiple sequence alignments. J.Mol.Biol. 237:182-192.

42. Sigrist, C.J., Cerutti, L., Hulo, N., Gattiker, A., Falquet, L., Pagni, M., Bairoch, A., and Bucher, P. 2002. PROSITE: a documented database using patterns and profiles as motif descriptors. Brief.Bioinform. 3:265-274.

43. Miyakawa, K., Ozawa, K., Uruno, T., and Imamura, T. 1999. The C-terminal region of fibroblast growth factor-1 is crucial for its biological activity and high level protein expression in mammalian cells. Growth Factors. 16:191-200.

44. Ron, D., Bottaro, D.P., Finch, P.W., Morris, D., Rubin, J.S., and Aaronson, S.A. 1993. Expression of biologically active recombinant keratinocyte growth factor. Structure/function analysis of amino-terminal truncation mutants. J.Biol.Chem. 268:2984-2988. 
45. Gerdes, J., Lemke, H., Baisch, H., Wacker, H.H., Schwab, U., and Stein, H. 1984. Cell cycle analysis of a cell proliferation-associated human nuclear antigen defined by the monoclonal antibody Ki-67. J.Immunol. 133:1710-1715.

46. Gerdes, J. 1990. Ki-67 and other proliferation markers useful for immunohistological diagnostic and prognostic evaluations in human malignancies. Semin.Cancer Biol. 1:199206.

47. Levin, R.M., Wein, A.J., Buttyan, R., Monson, F.C., and Longhurst, P.A. 1994. Update on bladder smooth-muscle physiology. World J Urol 12:226-232.

48. Baskin, L.S., Hayward, S.W., Sutherland, R.A., DiSandro, M.J., Thomson, A.A., Goodman, J., and Cunha, G.R. 1995. Mesenchymal-epithelial interactions in the bladder. World J Urol 14:301-309.

49. Baskin, L.S., Sutherland, R.S., Thomson, A.A., Nguyen, H.T., Morgan, D.M., Hayward, S.W., Hom, Y.K., DiSandro, M., and Cunha, G.R. 1997. Growth factors in bladder wound healing. J.Urol. 157:2388-2395.

50. Baskin,L.S., Hayward,S.W., DiSandro,M., Li,Y.W., and Cunha,G.R. 1999. Epithelialmesenchymal interactions in the bladder: Implications for bladder augmentation. In Advances in Bladder Research. L.S.Baskin and Hayward,S.W., editors. Kluwer Academic/Plenum, New York. 49-62.

51. Yi, E.S., Shabaik, A.S., Lacey, D.L., Bedoya, A.A., Yin, S., Housley, R.M., Danilenko, D.M., Benson, W., Cohen, A.M., and Pierce, G.F. 1995. Keratinocyte growth factor causes proliferation of urothelium in vivo. J.Urol. 154:1566-1570.

52. Ulich, T.R., Whitcomb, L., Tang, W., O'Conner, T.P., Tarpley, J., Yi, E.S., and Lacey, D. 1997. Keratinocyte growth factor ameliorates cyclophosphamide-induced ulcerative hemorrhagic cystitis. Cancer Res. 57:472-475.

53. Hines, M.D. and Allen-Hoffmann, B.L. 1996. Keratinocyte growth factor inhibits crosslinked envelope formation and nucleosomal fragmentation in cultured human keratinocytes. J.Biol.Chem. 271:6245-6251.

54. Igarashi, M., Finch, P.W., and Aaronson S.A. 1998. Characterization of recombinant human fibroblast growth factor (FGF)-10 reveals functional similarities with keratinocyte growth factor (FGF-7). J.Biol.Chem. 273:13230-13235.

55. Park, J.M., Yang, T., Arend, L.J., Smart, A.M., Schnermann, J.B., and Briggs, J.P. 1997. Cyclooxygenase-2 is expressed in bladder during fetal development and stimulated by outlet obstruction. Am J Physiol 273:F538-F544.

56. Santarosa, R., Colombel, M.C., Kaplan, S., Monson, F., Levin, R.M., and Buttyan, R. 1994. Hyperplasia and apoptosis. Opposing cellular processes that regulate the response of the rabbit bladder to transient outlet obstruction. Lab.Invest. 70:503-510. 
57. Karim, O.M., Seki, N., and Mostwin, J.L. 1992. Detrusor hyperplasia and expression of "immediate early" genes with onset of abnormal urodynamic parameters. Am.J.Physiol. 263:R1284-R1290.

58. Lu, W., Luo, Y., Kan, M., and McKeehan, W.L. 1999. Fibroblast growth factor-10. A second candidate stromal to epithelial cell andromedin in prostate. J.Biol.Chem. 274:1282712834.

59. Zhang, M.Z., Wen, J., Arakawa, T., and Prestrelski, S.J. 1995. A new strategy for enhancing the stability of lyophilized protein: the effect of the reconstitution medium on keratinocyte growth factor. Pharm.Res. 12:1447-1452.

60. Chen, B.L. and Arakawa, T. 1996. Stabilization of recombinant human keratinocyte growth factor by osmolytes and salts. J.Pharm.Sci. 85:419-426.

61. West, M.J., Slomianka, L., and Gundersen, H.J. 1991. Unbiased stereological estimation of the total number of neurons in thesubdivisions of the rat hippocampus using the optical fractionator. Anat.Rec. 231:482-497.

62. Charleston,J.S. 2003. Morphometric Analysis of Cell Number in the Central Nervous System. In Current Protocols in Toxicology . L.Costa, editor. John Wiley and Sons, Inc., New York.

63. Cochran,W.G. 1982. Sytematic Sampling. In Sampling Techniques, 3rd Edition. John Wiley \& Sons, New York. 205-232.

64. Charleston,J.S. 2003. Morphometric Analysis of Cell Number in the Central Nervous System. In Current Protocols in Toxicology. L.costa, editor. John Wiley and Son, Inc., New York.

65. West, M.J. and Slomianka, L. 1998. Total number of neurons in the layers of the human entorhinal cortex. Hippocampus 8:69-82.

66. Finch, P.W., Rubin, J.S., Miki, T., Ron, D., and Aaronson, S.A. 1989. Human KGF is FGFrelated with properties of a paracrine effector of epithelial cell growth. Science 245:752-755.

67. Zhang, M.Z., Wen, J., Arakawa, T., and Prestrelski, S.J. 1995. A new strategy for enhancing the stability of lyophilized protein: the effect of the reconstitution medium on keratinocyte growth factor. Pharm.Res. 12:1447-1452. 
Table 1. Distribution of the Sequence for FGF-7 in the Human Genome ${ }^{a}$

\begin{tabular}{|c|c|c|c|c|}
\hline Chromosome & $\begin{array}{l}\text { Begin - End of } \\
\text { Genomic Contig }^{b}\end{array}$ & $\begin{array}{l}\text { Accession \# } \\
\text { of Contig }\end{array}$ & $\begin{array}{l}\text { Begin - End of } \\
\text { cDNA }\end{array}$ & $\begin{array}{l}\text { \% Identity } \\
\text { (Length) }\end{array}$ \\
\hline \multirow[t]{2}{*}{9} & 310522 - 313542 & NT_033211.3 & $834-3853$ & 97 (3032) \\
\hline & 309333 - 309436 & & $732-835$ & $97(105)$ \\
\hline \multirow[t]{3}{*}{9} & $205822-202808^{c}$ & NT_033210.2 & $834-3853$ & $96(3033)$ \\
\hline & 112194-110283 & & $19 \overline{4} 1-3853$ & $96(1921)$ \\
\hline & $207019-206916^{c}$ & & $732-835$ & $97(104)$ \\
\hline \multirow[t]{4}{*}{15} & $4748306-4751324$ & NT_010194.13 & $834-3853$ & $99(3021)$ \\
\hline & $4688024-4688581$ & & $174-731$ & $99(558)$ \\
\hline & 4687260 & & $1=179$ & $100(179)$ \\
\hline & $4747149-4747252$ & & $732-835$ & $100(104)$ \\
\hline \multirow[t]{2}{*}{18} & $60149-57146^{c}$ & NT_024983.8 & $834-3853$ & $95(3030)$ \\
\hline & $61341-61238^{c}$ & & $732-835$ & $96(194)$ \\
\hline \multirow[t]{2}{*}{21} & $363464-366469$ & NT_011512.7 & $834-3824$ & 96 (3029) \\
\hline & $362274-362377$ & & $732-835$ & $97(104)$ \\
\hline
\end{tabular}

${ }^{\text {a}}$ Human genome database was queried with the cDNA sequence for bladder FGF-7 and with the NCBI reference sequence for the full-length FGF-7 mRNA (RefSeq Accession NM_002009.2). Dotted lines bracket genomic sequences found with the reference sequence, but not with the bladder sequence bunits in kbp; ' sequence found on minus strand (italicized type). 
Table 2. Intron / Exon Structure of Human FGF-7 Gene on Chromosome 15

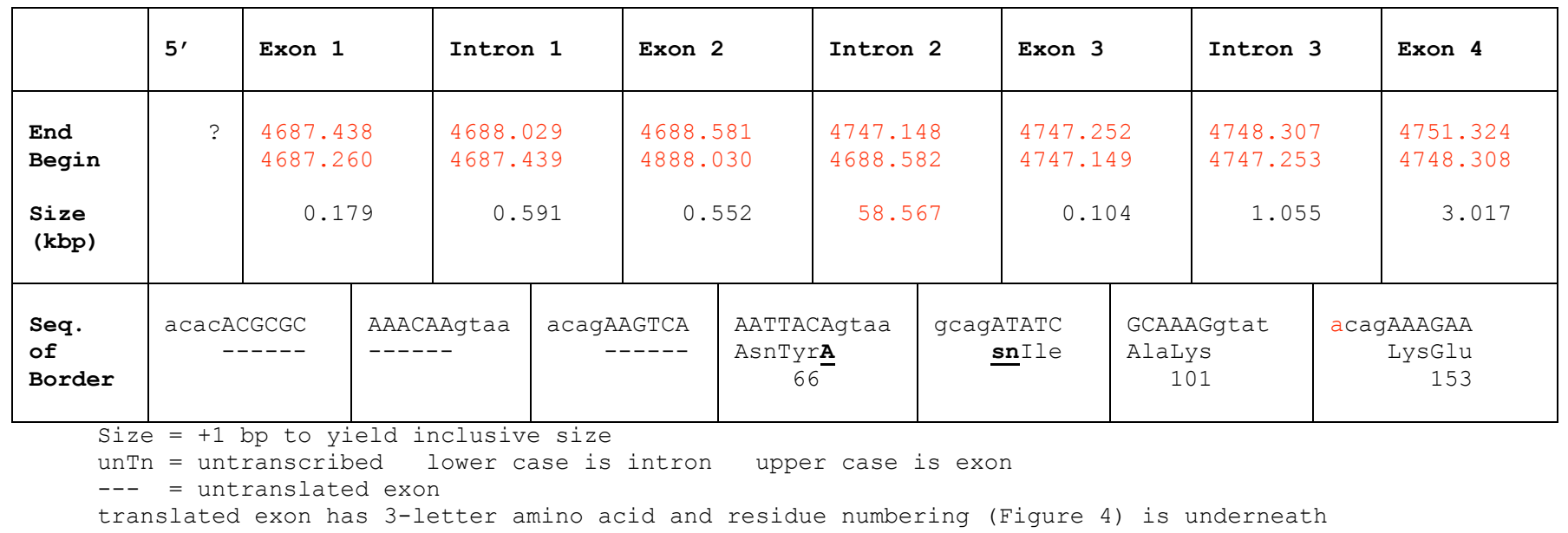


Table 3. Distribution of Chromosome 15 FGF-7 Sequences on Other Chromosomes ${ }^{\mathrm{a}}$

\begin{tabular}{|c|c|c|c|c|c|c|c|c|}
\hline & $\begin{array}{l}\text { Chr 15 } \\
\text { (length in } \\
\text { bp) }\end{array}$ & $\begin{array}{l}\text { Chr } 18 \\
\text { (length in } \\
\text { bp) }\end{array}$ & $\begin{array}{l}\text { Chr } 21 \\
\text { (length in } \\
\text { bp) }\end{array}$ & $\begin{array}{l}\text { Chr 9pt } \\
\text { (length in bp) }\end{array}$ & $\begin{array}{l}\text { Chr 9pm } \\
\text { (length in bp) }\end{array}$ & $\begin{array}{l}\text { Chr 9pc } \\
\text { (length in bp) }\end{array}$ & $\begin{array}{l}\text { Chr 9qc } \\
\text { (length in } \\
\text { bp) }\end{array}$ & $\begin{array}{l}\text { Chr 9qt } \\
\text { (length in bp) }\end{array}$ \\
\hline $\begin{array}{l}\mathrm{CNS}^{\mathrm{b}} \text { of Intron } \\
2\end{array}$ & $\begin{array}{l}47,343,436- \\
47,354,485 \\
(11,050)\end{array}$ & $\begin{array}{l}14878089- \\
14889070 \\
(10.982)\end{array}$ & $\begin{array}{l}13636398- \\
13643456 \\
(7,059)\end{array}$ & Gap & $\begin{array}{l}41146340- \\
41152725 \\
(6,372)\end{array}$ & $\begin{array}{l}41867144- \\
41870812 \\
(3,669) \text { plus } \\
\text { Gap }\end{array}$ & $\begin{array}{l}61209613- \\
61214951 \\
(5,339)\end{array}$ & $\begin{array}{l}\text { 62207569- } \\
62213939 \\
(6,371)\end{array}$ \\
\hline $\begin{array}{l}\text { Exon } 3- \\
\text { translated }\end{array}$ & $\begin{array}{l}47,354,486- \\
47,354,589 \\
(104)\end{array}$ & $\begin{array}{l}14877985- \\
14878088 \\
(104)\end{array}$ & $\begin{array}{l}13643457- \\
13643560 \\
(104)\end{array}$ & Gap & $\begin{array}{l}41146236- \\
41146339 \\
(104)\end{array}$ & Gap & $\begin{array}{l}\text { 61214952- } \\
61215055 \\
(104)\end{array}$ & $\begin{array}{l}\text { 62213940- } \\
62214043 \\
(104)\end{array}$ \\
\hline CNS of Intron 3 & $\begin{array}{l}47,354,590- \\
47,355,644 \\
(1,055)\end{array}$ & $\begin{array}{l}14876895- \\
14877984 \\
(1,090)\end{array}$ & $\begin{array}{l}13643561- \\
13644648 \\
(1,088)\end{array}$ & Gap & $\begin{array}{l}41145149- \\
41146235 \\
(1,087)\end{array}$ & Gap & $\begin{array}{l}61215056- \\
61216150 \\
(1,095)\end{array}$ & $\begin{array}{l}62214044- \\
62215130 \\
(1,087)\end{array}$ \\
\hline $\begin{array}{l}\text { Exon 4- } \\
\text { translated }\end{array}$ & $\begin{array}{l}47,355,645- \\
47,355,839 \\
(195)\end{array}$ & $\begin{array}{l}14876701- \\
14876894 \\
(194)\end{array}$ & $\begin{array}{l}13644649- \\
13644840 \\
(192)\end{array}$ & Gap & $\begin{array}{l}41144954- \\
41145148 \\
(195)\end{array}$ & Gap & $\begin{array}{l}\text { 61216151- } \\
61216346 \\
(196)\end{array}$ & $\begin{array}{l}62215131- \\
62215325 \\
(195)\end{array}$ \\
\hline Exon 4 - UTR & $\begin{array}{l}47,355,840- \\
47,358,659 \\
(2,820)\end{array}$ & $\begin{array}{l}14873895- \\
14876700 \\
(2,806)\end{array}$ & $\begin{array}{l}13644841- \\
13647650 \\
(2,810)\end{array}$ & $\begin{array}{l}\text { 41106274- } \\
41107090 \\
\text { (817) plus } \\
\text { Gap }\end{array}$ & $\begin{array}{l}41142132- \\
41144953 \\
(2,820)\end{array}$ & Gap & $\begin{array}{l}\text { 61216347- } \\
61219161 \\
(2,815)\end{array}$ & $\begin{array}{l}62215326- \\
62218147 \\
(2,822)\end{array}$ \\
\hline $\begin{array}{l}\text { CNS of 3' } \\
\text { region }\end{array}$ & $\begin{array}{l}47,358,660- \\
47,361,636 \\
(2,977)\end{array}$ & $\begin{array}{l}14870961- \\
14873894 \\
(2,934)\end{array}$ & $\begin{array}{l}13647651- \\
13650563 \\
(2,913)\end{array}$ & $\begin{array}{l}41107091- \\
41110060 \\
(2,970)\end{array}$ & $\begin{array}{l}\text { 41139214- } \\
41142131 \\
(2,918)\end{array}$ & Gap & $\begin{array}{l}\text { 61219162- } \\
61220136 \\
(975)\end{array}$ & $\begin{array}{l}\text { 62218148- } \\
62218307 \\
(160) \text { plus } \\
\text { Gap }\end{array}$ \\
\hline
\end{tabular}

${ }^{a}$ Sequence Data of the UCSC Genome Browser on April 2003 Freeze

${ }^{\mathrm{b}}$ Conserved Non-coding Sequences 
Table 4. Quantification of Urothelial Cell Number by Stereology

\begin{tabular}{|c|c|c|c|c|}
\hline & $\begin{array}{l}\text { Total \# urothelial } \\
\text { cells } \times 10^{6} \\
\text { (mean } \pm \text { S.E.M.) }\end{array}$ & $\begin{array}{c}\text { Total \# Ki67 } \\
\text { positive } \\
\text { urothelial cells } \\
\qquad \times 10^{4} \\
\text { (mean } \pm \text { S.E.M.) }\end{array}$ & $\begin{array}{c}\% \mathrm{Ki}-67 \\
\text { positive cells } \\
\text { (mean } \pm \text { S.E.M.) }\end{array}$ & $\begin{array}{l}\text { Total \# fibroblasts } \\
\text { positive for FGF-10 } \\
\times 10^{3} \\
\text { (mean } \pm \text { S.E.M.) }\end{array}$ \\
\hline WT $(n=8)$ & $0.81 \pm 0.13$ & $1.90 \pm 1.22$ & $1.78 \pm 0.46$ & $0.20 \pm 0.06$ \\
\hline $\mathrm{KO}(\mathrm{n}=6)$ & $1.31 \pm 0.26$ & $2.02 \pm 0.36$ & $1.60 \pm 0.94$ & $1.09 \pm 0.38$ \\
\hline
\end{tabular}




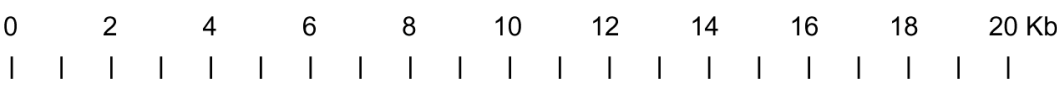

E3 E4

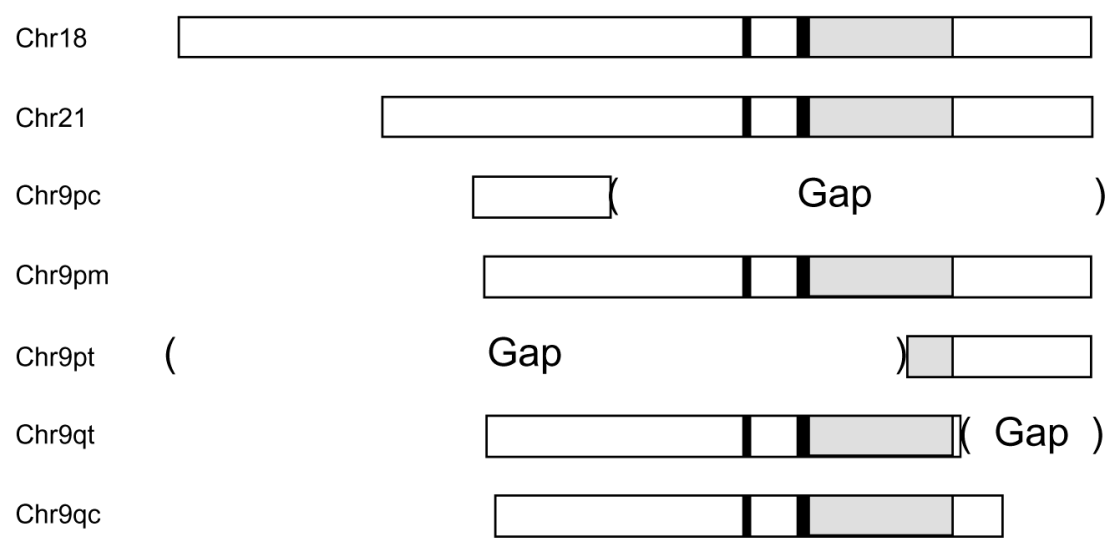

Figure 1. Duplication of FGF-7 sequences among human chromosomes. The April 2003 version of the Human Genome Database was analyzed for the presence of chromosome 15 FGF-7 gene elements. Black boxes, coding exons (E3 and E4). Stippled boxes, 3' untranslated regions; White boxes, introns and 3' flanking sequences. Chr9pc, FGF-7 copy located proximal to the centromere on the $\mathrm{p}$ arm. Chr9pm, middle copy on the $\mathrm{p}$ arm. Chr9pt, copy located proximal to the telomere on the $p$ arm. Chr9qt, copy located proximal to the telomere on the $q$ arm. Chr9qc, copy located proximal to the centromere on the $\mathrm{q}$ arm. 


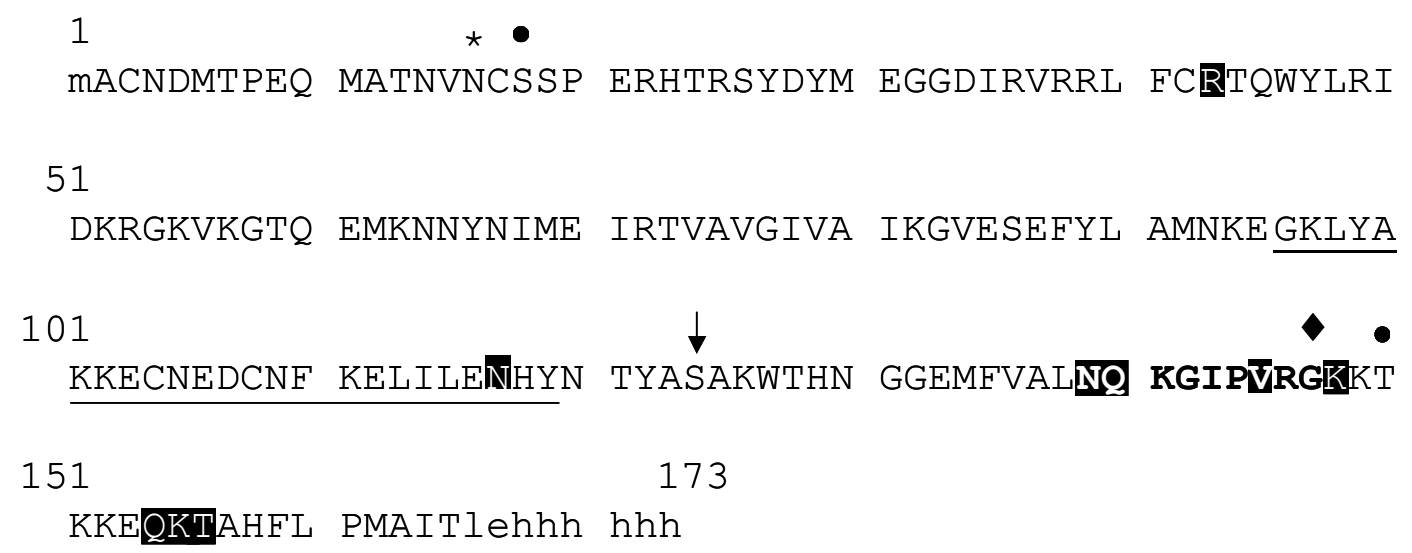

Figure 2. Sequence analysis of human urinary bladder FGF-7 and primary structure of rFGF7-His. The amino acid sequence for rFGF7-His was deduced from data obtained by the dideoxy sequencing of the PCR-DNA product displayed in Figure 1, lane 2. Residues of rFGF7-His that are not part of the 164 aa wild-type sequence (uppercase) are represented in lowercase and include the formyl-methionine (residue 1) and Leu, Glu, and six His residues (residues 166-173). Diamond indicates site of putative amidation (Gly147). Asterisk indicates site of N-linked glycosylation (Asn16). Filled circles indicate sites of casein kinase II phosphorylation (Ser18 and Thr150). Arrow indicates sites of phosphorylation by protein kinase C (Ser124). Underlined residues represent the FGF family signature (Gly96-Tyr119) (Prosite Database). Bolded residues represent the glycine box (Asn139-Gly147). Black boxes with white letters designate residues implicated in the heparin-binding motif (Arg43, Asn117, Asn139, Gln140, Val145, Lys148, Asn154, Lys155, and Thr156). 


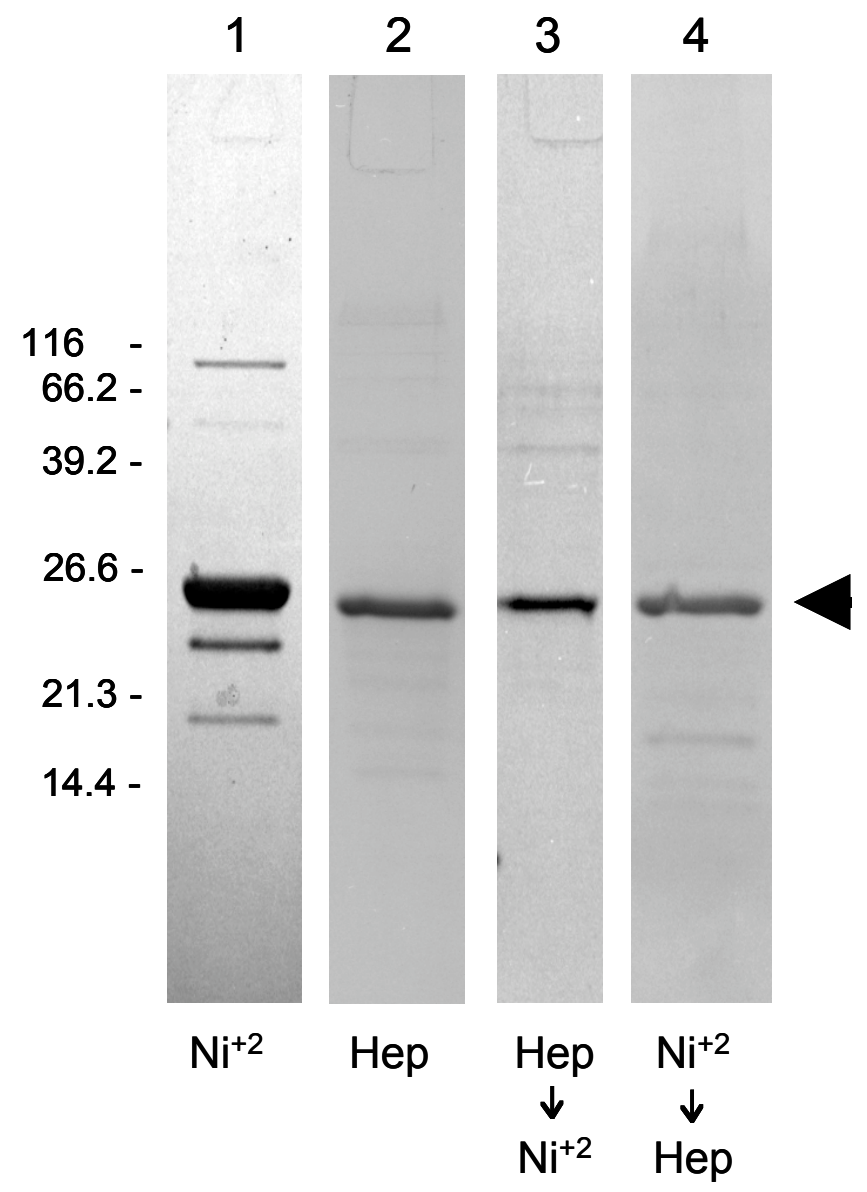

Figure 3. Interaction of rFGF7-His with metal-chelate and heparin affinity chromatographies. Shown are composite SDS-containing electrophoretic gels stained with GelCode Blue. Lane 1, elution from Ni-NTA resin with pH 5.3 buffer. Lane 2, elution from heparin-affinity column with $1.0 \mathrm{M} \mathrm{LiCl}$. Lane 3, 1.0 M elution product from heparinaffinity resin was applied to a Ni-NTA resin. Shown is the elution of rFGF7-His from the Ni-NTA resin with pH 5.3 buffer. Lane 4, pH 5.3 elution product from Ni-NTA resin was applied to a heparin-affinity column. Shown is the elution of rFGF7-His from the heparinaffinity resin with $1.0 \mathrm{M} \mathrm{LiCl}$. 

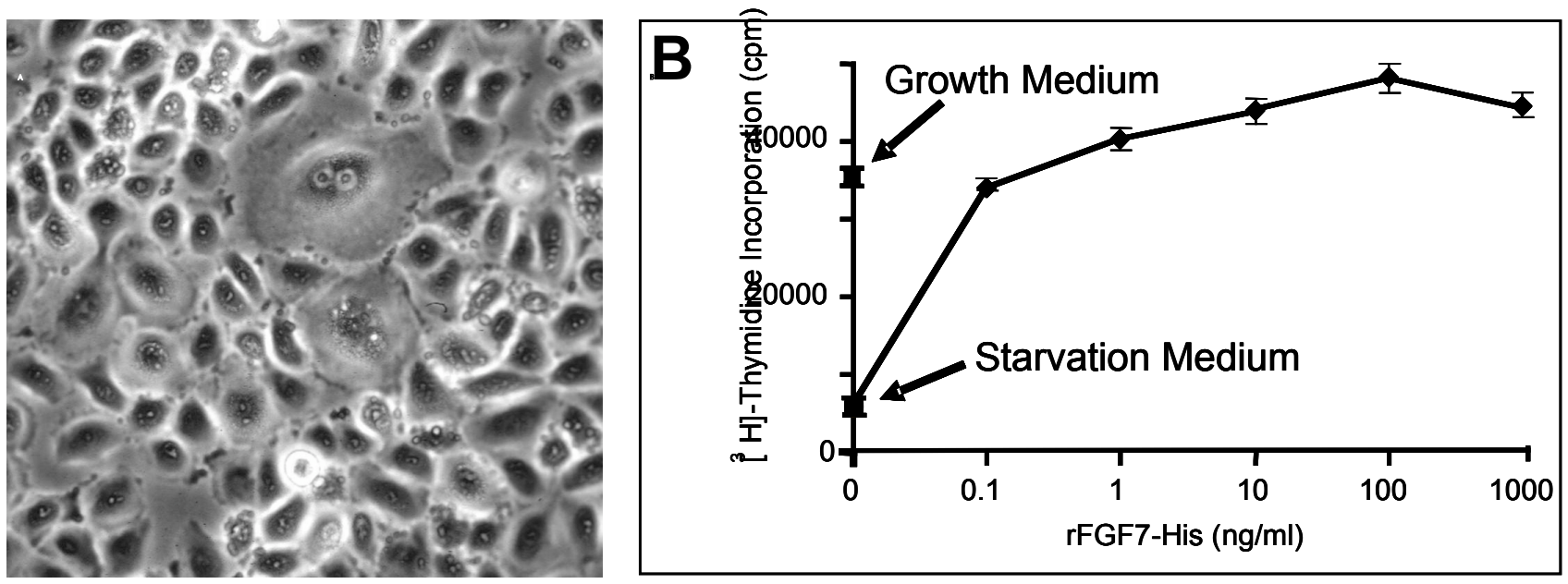

Figure 4. Mitogenic activity of rFGF7-His on urothelial cell DNA synthesis. $A$, Primary culture of human urothelial cells at confluency (passage 3). Note large supeficial cell at top of photograph and the round, refractile dividing basal cell at bottom. B, Passage 4 bladder urothelial cells were plated at a density of 39,000 cells well $^{-1}$ of a 96-well plate and grown to confluency in the presence of growth medium. Cultures were rendered quiescent by incubation for $16 \mathrm{hr}$ in medium that lacked growth stimulators ("starvation medium"). Increasing concentrations of rFGF7-His were mixed with starvation medium that contained $0.5 \mu \mathrm{Ci} \mathrm{ml} l^{-1}\left[{ }^{3} \mathrm{H}\right]$-thymidine added in a pulse-chase experiment. 


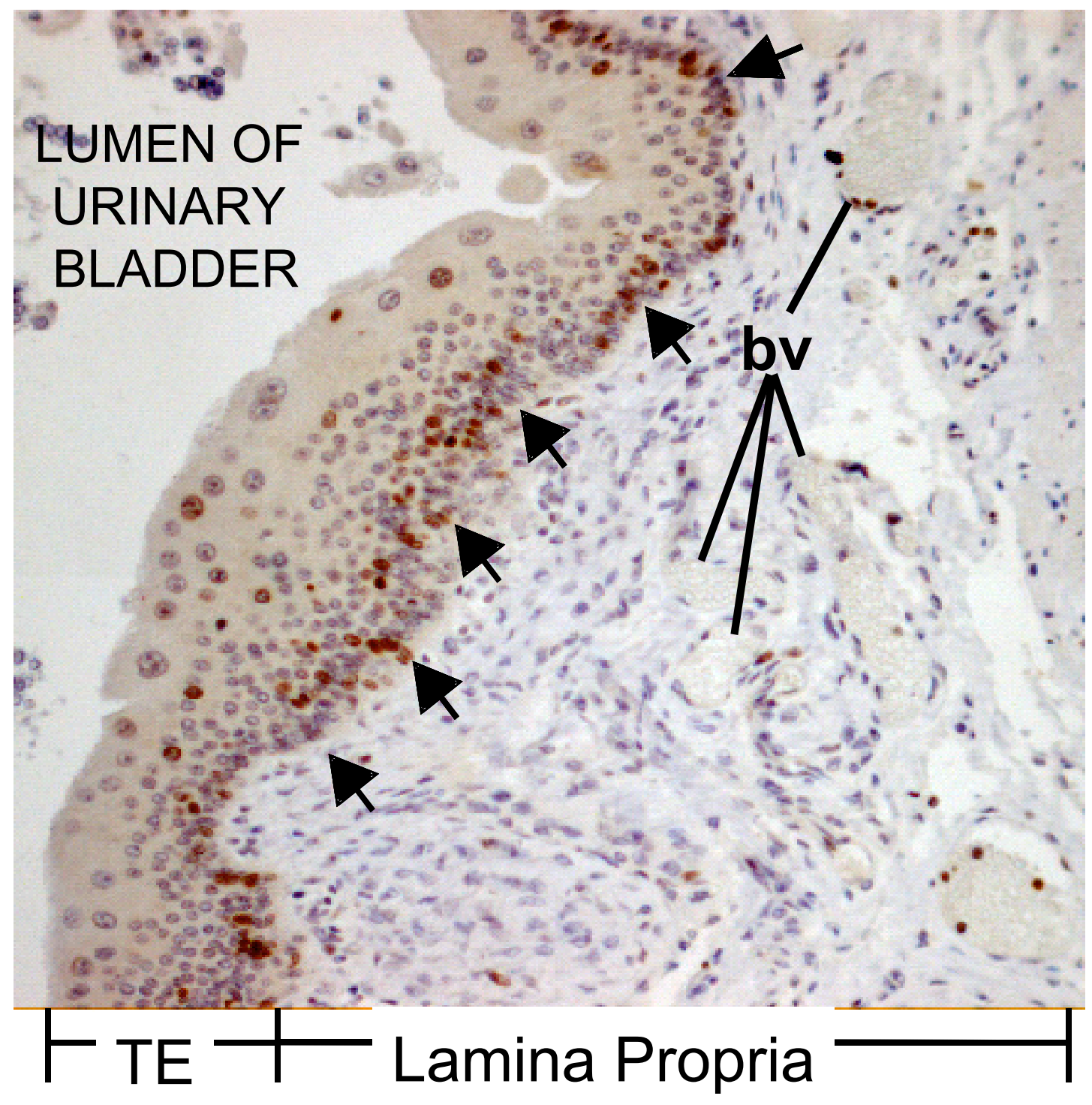

Figure 5. Induction of murine urothelial cell proliferation by recombinant FGF-7 (Palifermin). rFGF-7 (Palifermin) was administered via intraperitoneal injection each day for 14 days to C57BL/6J mice. Shown is a section of urinary bladder mucosa that reacts with immunoglobulins specific for the nuclear antigen Ki-67. Arrows, regions of urothelium that contain cells positive for Ki-67 (brown nuclei). bv, capillaries. Counterstained with toluidine blue. 200x. 

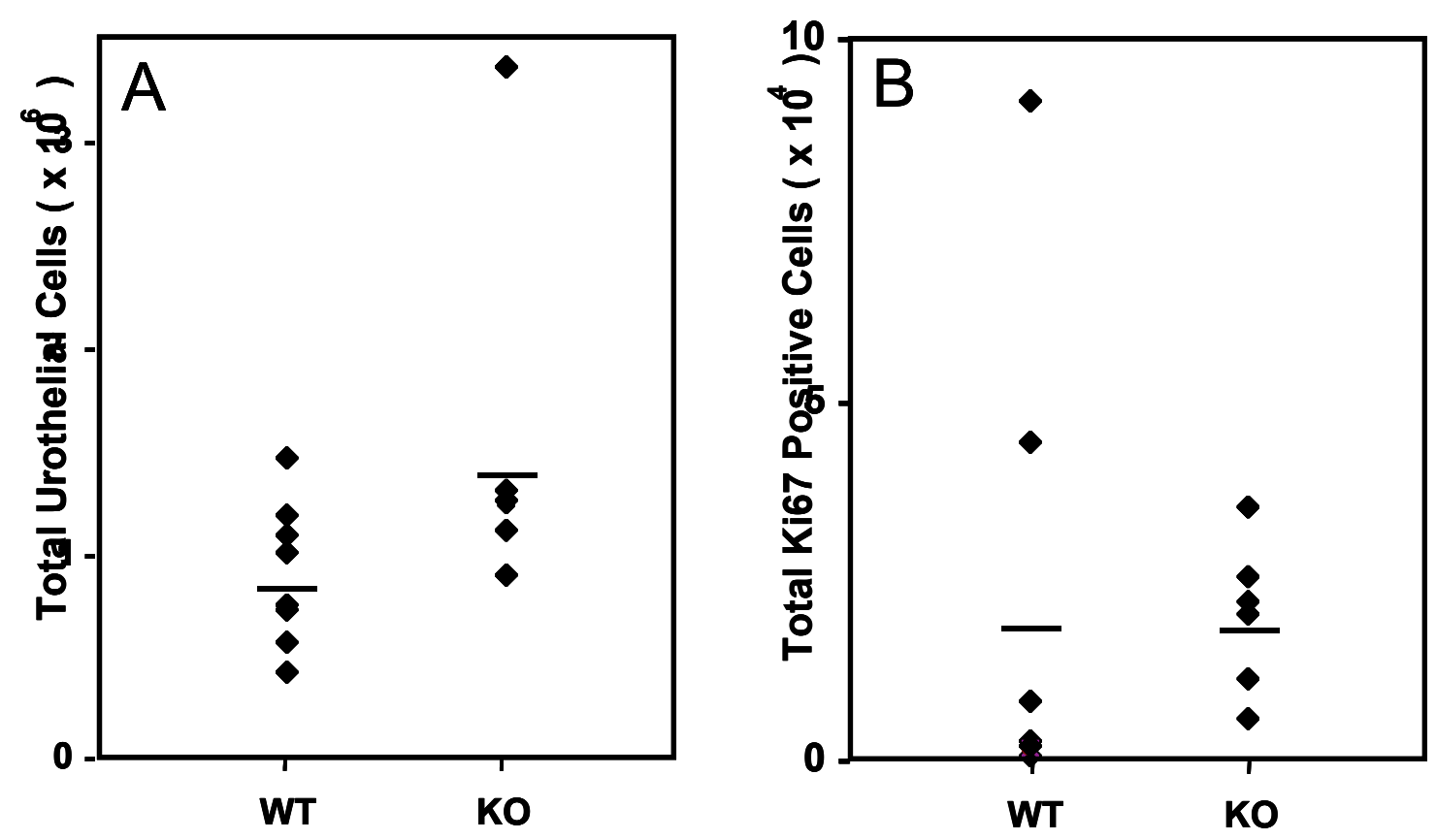

Figure 6. Proliferative response of the murine urothelium in response to partial outlet obstruction. Wild-type or FGF7-null mice (C57BL/6J strain, 8-weeks old) were anesthetized and the urogenital cavity was opened. A $26 \mathrm{~g}$ catheter was inserted into the urethra and up into the bladder. Silk-thread was tied around the urethra-bladder neck junction. The catheter was removed and reinserted to verify that partial outlet obstruction would result. After closure of the cavity, mice were allowed to recover, and housed for 8 additional days. After animals were euthanized, bladders were removed, fixed, and paraffin-embedded. Sections were cut according to principles of unbiased stereology, immunostained with antibodies specific for the proliferation marker Ki-67, and counterstained with Toluidine blue. Quantification of total number of urothelial cells (A) and total Ki-67 positive urothelial cells (B) was achieved by stereological counting (64). Reliable estimates of the number of Ki-67 positive proliferating cells contained within the developing urinary tract was determined by use of the Optical Fractionator $(61,65)$. wt (wildtype), ko (knockout or FGF7-null), horizontal bar is mean. Diamond, cell count for an individual animal that underwent urethral ligation surgery. See Table 4 for corresponding values. 


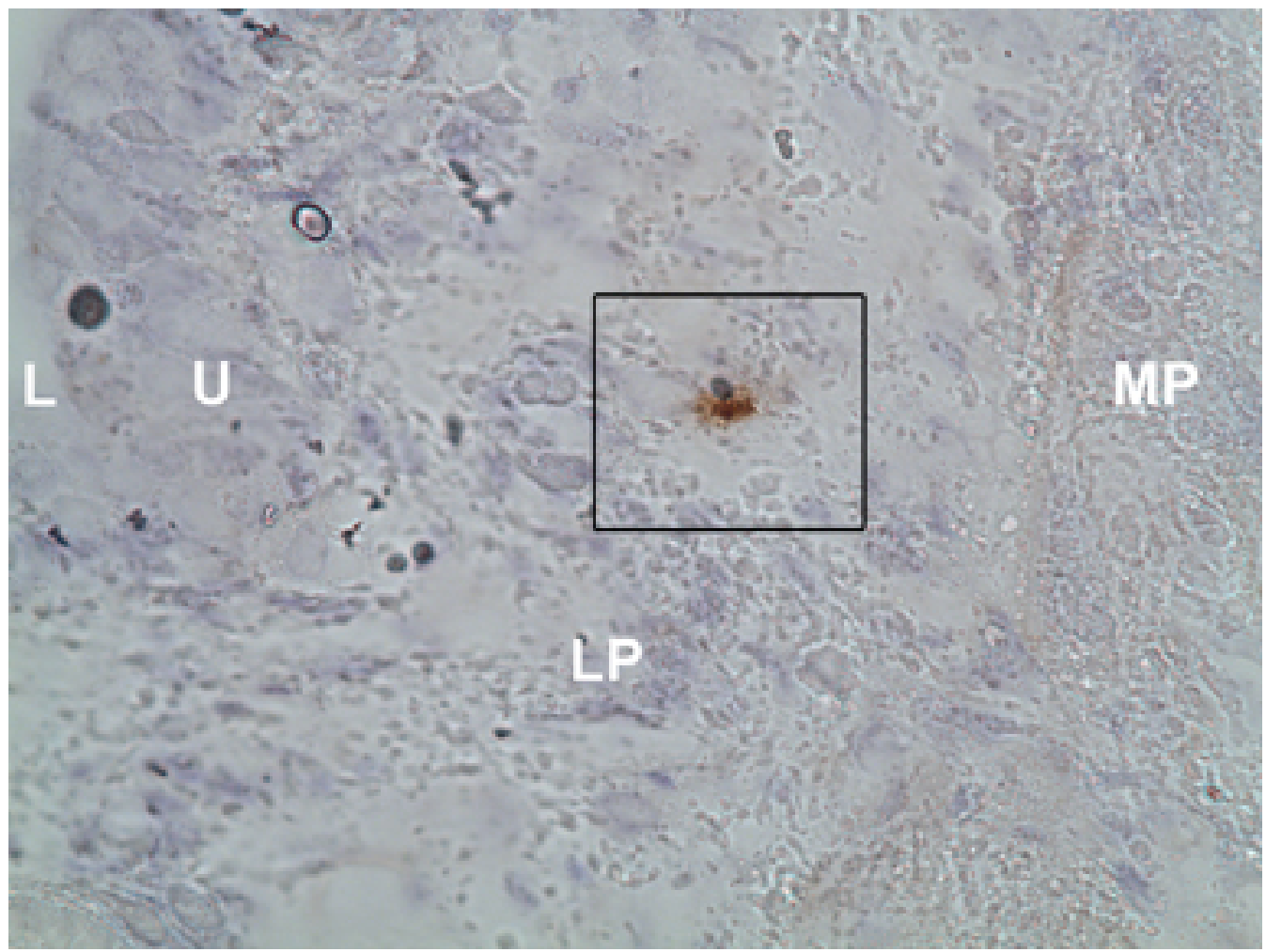

Figure 7. Representative disector placement on FGF-10 positive lamina propria fibroblast cell. Shown is a representative section from a FGF7-null mouse bladder immunostained with monoclonal antibodies specific for FGF-10. Rectangle, disector; L, lumen; U, urothelium; LP, lamina propria; MP, muscularis propria. Size bar $=\quad \mu \mathrm{m}$. 


\section{SUPPLEMENTAL MATERIALS SECTION}

Materials and Methods: Materials

Human urinary bladder cDNA was obtained from Invitrogen (Carlsbad, CA).

Oligonucleotide primers were synthesized by Keystone Laboratories (Foster City, CA).

Escherichia coli strains NovaBlue and BL21trxB(DE3), Perfectly Blunt Cloning Kit, plasmid

pET21d, Bug Buster lysis reagent, and carbenicillin were purchased from Novagen (Madison,

WI). Restriction enzymes NcoI and XhoI, T4 DNA ligase, Taq polymerase, and Complete

Protease Inhibitors were from Roche Molecular Biochemicals (Indianapolis, IN). Ni-NTA metalchelate affinity resin was from Qiagen (Chatsworth, CA). HiTrap heparin-sepharose affinity resins were from Amersham Biochemicals (Piscataway, NJ). SDS-PAGE gels and the Amplified Alkaline Phosphatase ImmunoBlot Assay detection system were obtained from BioRad (Hercules, CA). Tetracycline and kanamycin were from Calbiochem (La Jolla, CA).

Recombinant FGF-7 (rFGF-7, Palifermin, rHuKGF)) was provided by Amgen (Thousand Oaks, CA). Rabbit anti-bovine uroplakin immunoglobulins were obtained from Dr. H. Sun (New York University, New York, NY).

\section{Methods: Isolation and Dideoxy Sequencing of FGF-7 from Human Urinary Bladder}

A population of human bladder cDNAs was used as a template for Taq polymerization. One pair of oligonucleotide primers was synthesized: "NcoI", 5'CATCCGGATCCATGGCTTGCAATGACATGACTCCA-3” and “XhoI”, 5’ACTCTCCGGCTACTCGAGAGTTATTGCCATAGGAAG-3'. These primers were designed to partially anneal to FGF-7 cDNA - the noncomplimentary regions encoded sites for the restriction endonucleases $\mathrm{NcoI}$ and $\mathrm{XhoI}$. Initial PCR conditions employed were 1 cycle at $95{ }^{\circ} \mathrm{C}$ 
for $12 \mathrm{~min}, 30$ cycles at $95^{\circ} \mathrm{C}$ and $60{ }^{\circ} \mathrm{C}$ at $30 \mathrm{~s}$ each, 1 cycle at $72{ }^{\circ} \mathrm{C}$ for $10 \mathrm{~min}$, and a hold at 4 ${ }^{\circ} \mathrm{C}$. Subsequent gel electrophoresis indicated a lack of detectable PCR-DNA product. Forty $\mu 1$ of this reaction product was used as template in a second round of Taq polymerization with the following thermocycler parameters: 1 cycle at $95^{\circ} \mathrm{C}$ for $12 \mathrm{~min}, 40$ cycles at $95^{\circ} \mathrm{C}$ and $50{ }^{\circ} \mathrm{C}$ and $72{ }^{\circ} \mathrm{C}$ for $1 \mathrm{~min}$ each, 1 cycle at $72{ }^{\circ} \mathrm{C}$ for $12 \mathrm{~min}$, and a hold at $4{ }^{\circ} \mathrm{C}$. Following gel electrophoresis, $250 \mathrm{ng}$ of the principal PCR-DNA product at $0.52 \mathrm{kbp}$ was excised from the gel, recovered by extraction, converted to blunt ends, ligated into pSTBlue-1, and transformed into competent NovaBlue E. coli. Subsequent growth at $37^{\circ} \mathrm{C}$ on agar plates that contained Luria broth, $50 \mu \mathrm{g} \mathrm{ml}^{-1}$ carbenicillin, and $15 \mu \mathrm{g} \mathrm{ml}^{-1}$ kanamycin, resulted in recombinant cultures that were archived as glycerol stocks and characterized with respect to insert size and dideoxy DNA sequencing. Clones that were identified as containing correctly oriented and in-frame inserts were designated pSTBlue-FGF7.

\section{Methods: Cloning into the Bacterial Expression Vector pET21d}

Six and one-half $\mu \mathrm{g}$ of pSTBlue-FGF7 were restricted with $\mathrm{NcoI}$ and $\mathrm{XhoI}$ endonucleases and electrophoresed through an agarose gel. The DNA of a $0.52 \mathrm{kbp}$ band was excised from the gel, extracted, and ligated to the expression plasmid pET21d, previously prepared by incubation with NcoI and XhoI and dephosphorylation with shrimp alkaline phosphatase. After transformation into BL21trxB(DE3) E. coli, recombinant colonies were generated by growth on Luria broth-agar plates that contained $50 \mu \mathrm{g} \mathrm{ml}^{-1}$ carbenicillin, stored as glycerol stocks, and designated pET21dFGF7. The resultant open reading frame that included six C-terminal His residues generated a fusion protein designated as $\mathrm{rFGF7-His.}$ 


\section{Methods: Expression, Isolation, and Characterization of Recombinant FGF-7 in E. coli}

The insert coding region from pSTBlue1-FGF7 was recloned into the pET21d expression vector to ultimately form the expression plasmid pET21d-FGF7. The expression of recombinant FGF-7 (rFGF-7) by transformed E. coli was found to be dependent on the addition of isopropyl1-thio- $\beta$-D-galactopyranoside, a reagent known to inactivate the lac repressor, thereby permitting synthesis of FGF-7 mRNA.

\section{Methods: Stereology Procedures}

Estimates of the number of total and proliferating (Ki-67 positive) urothelial cells of obstructed murine bladders were determined by use of the Optical Fractionator (61). The Optical Fractionator method combines a 3-dimensional unbiased counting frame (the optical disector) and an unbiased sampling method (the fractionator) to generate estimates of the total number of particles of interest, in this case Ki-67 positive urothelial cells. The ensuing estimate is unbiased and free of volume artifacts commonly present with other counting methods (62). Implementation of the Optical Fractionator was performed using a BH-2 Olympus microscope equipped with an Optronics DEI 750 digital video camera and Ludl high precision motorized stage. Data collection and microscope operation was controlled by Stereo Investigator software (version 3.19, MicroBrightField, Williston, Vermont).

The application of the fractionator in the present sampling design consisted of initially systematically collecting every $40^{\text {th }}$ section across the entire bladder starting with a random start (e.g. a random start might be 12 , therefore collect the $12,52,92 \ldots$ sections). Hence this set of sections (and the structure of interest contained within, i.e., the obstructed bladder) represented $1 / 40^{\text {th }}$ of the entire structure. Following staining of this set of sections, Ki-67 positive urothelial 
cells were counted in a known aerial fraction of the transitional epithelial layer. The aerial fraction was determined by placing a random stepping grid, generated by the Stereo Investigator software program, over the section. An appropriate stepping grid (500 x $500 \mu \mathrm{m}$, or an area of $250,000 \mu^{2}$ ) was used to place sequential disectors across the surface of the sections. A disector frame $\left(10 \times 10=100 \mu \mathrm{m}^{2}\right)$ was placed in each stepping grid and used to count Ki-67 positive cells according to optical disector counting rules. Immunopositive cells were readily identified by the dark brown coloration of the nuclei characteristic of the staining procedures (see Figure 5 in manuscript). The overlay of this stepping grid was placed randomly (independently) over the transitional epithelium, thus ensuring that each step was systematic and random. In our experiments, the optical disectors sampled $1 / 2500^{\text {th }}$ of the transitional epithelium. Combined with the $1 / 40^{\text {th }}$ of the structure represented by the section, the optical disectors sampled $1 / 100,000^{\text {th }}$ of the entire structure. Cell counts were summed from all disectors across all sections collected, and the total number was determined by multiplying the number counted by the inverse of the fraction sampled. Systematic random sampling has been determined to be fair because every part of the original structure had an equal probability of being selected due to the random position set within the first interval, and systematic random sampling offers additional advantages of efficiency (63).

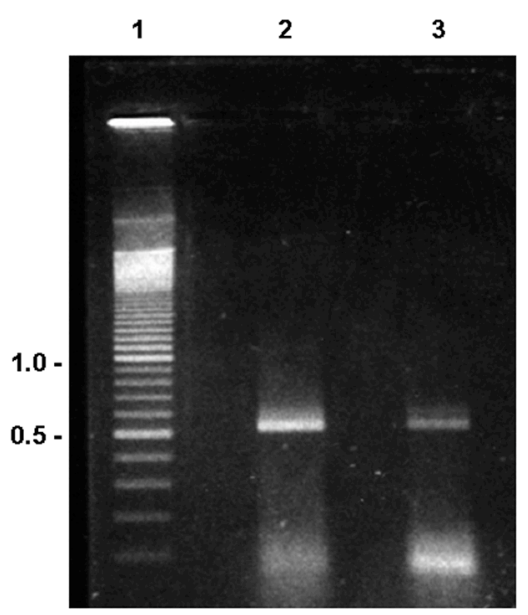

Supplemental Figure 1. Agarose gel electrophoresis of human urinary bladder FGF-7 cDNA.

Lane 1,100-bp markers are indicated to the left of the figure in $\mathrm{kbp}$

Lane 2, RT-PCR DNA products encoding FGF-7 from oligonucleotide primers designed to generate rFGF7-His. Lane 3, RT-PCR DNA products encoding FGF-7 from oligonucleotide primers designed to generate wild-type rFGF-7. Shown is a fluorescent image generated bv nhotogranhv of the 


\section{Results: Isolation and Sequence Analysis of FGF-7 cDNA from Human Urinary Bladder}

A partial cDNA fragment that encoded the mature, secreted form of FGF-7 was isolated from a human urinary bladder cDNA library by reverse transcription (RT) and PCR. Supplemental Figure 1 demonstrates that the RT-PCR yielded a product that was approximately $0.52 \mathrm{kbp}$ in size, in agreement with the size predicted from the FGF-7 cDNA cloned from the human embryonic lung cell line M426 (Accession M60828) (66).

The $0.52 \mathrm{kbp}$ RT-PCR product was subcloned into the high copy vector pSTBlue1 to form the recombinant plasmid designated pSTBlue1-FGF7. Subsequent dideoxy sequencing of this $0.52 \mathrm{kbp}$ RT-PCR product revealed the presence of a $0.492 \mathrm{kbp}$ bladder sequence. This sequence was found to be $100 \%$ identical with the corresponding region present in the NCBI reference sequence encoding FGF-7 mRNA (Accession NM_002009.2).

\section{Results: Expression of Recombinant FGF7-His in E. coli}

Two types of recombinant (r) FGF-7 were used in this study. rFGF-7 (lacking a C-terminal His-tag) was prepared and provided by Amgen as the pharmaceutical Palifermin (67). rFGF7-His was prepared as described below.

A loop of recombinant bacteria from a frozen glycerol stock was streaked on a plate of agar that contained Media A (Luria broth, $100 \mu \mathrm{g} \mathrm{ml}^{-1}$ carbenicillin, and $0.2 \%$ glucose) and incubated at $37{ }^{\circ} \mathrm{C}$ for $16-24 \mathrm{hr}$. A $50 \mathrm{ml}$ starter culture in Media A was inoculated with a single colony and propagated until the optical density at $600 \mathrm{~nm}$ reached $\sim 0.6$. After storage of the culture overnight at $4{ }^{\circ} \mathrm{C}$, cells were recovered by sedimentation at $5000 \mathrm{x}$ g, resuspended in $4 \mathrm{ml}$ of Media A, and inoculated into $2 \mathrm{~L}$ of prewarmed Media A. Once the cultures reached an optical density at $600 \mathrm{~nm}$ of $\sim 0.6$, isopropyl-1-thio- $\beta$-D-galactopyranoside was added to a final 
concentration of $1 \mathrm{mM}$. After 3-4 hrs of continued growth at $37^{\circ} \mathrm{C}$, cells were collected by sedimentation at $5,000 \mathrm{x}$ g at $4{ }^{\circ} \mathrm{C}$ and frozen at $-20{ }^{\circ} \mathrm{C}$.

Luria broth used in these experiments contained peptone derived from meat so as to eliminate lactose that would normally be found in peptone derived from milk. We have found that lactose in the medium elicits premature activation of the T7lac promoter and of rFGF7-His synthesis.

\section{Isolation of rFGF7-His}

Thawed pET21d-FGF7-BL21trxB(DE3) cells were lysed with Bug Buster reagent that contained additional Complete protease inhibitors. Lysed material was fractionated into soluble and insoluble fractions by centrifugation at $20,000 \mathrm{x}$ for $45 \mathrm{~min}$ at $4{ }^{\circ} \mathrm{C}$.

Soluble material was dialyzed overnight at $4{ }^{\circ} \mathrm{C}$ versus Buffer A $\left(0.1 \mathrm{M} \mathrm{NaH}_{2} \mathrm{PO}_{4}, 0.3 \mathrm{M}\right.$ $\mathrm{NaCl}$, and $0.05 \mathrm{M}$ Tris- $\mathrm{HCl})(\mathrm{pH} 8.0)$. After clarification of the dialyzate by centrifugation at $20,000 \mathrm{x}$ g for $20 \mathrm{~min}$ at $4{ }^{\circ} \mathrm{C}$, imidazole was added to the soluble phase at a final concentration of $0.025 \mathrm{M}$. This sample mixture was then applied to a $4 \mathrm{ml}$ column of Ni-NTA resin previously equilibrated in Buffer A ( $\mathrm{pH}$ 8.0). The column was developed with Buffer A (pH 8.0) until the $\mathrm{A}_{280 \mathrm{~nm}}<0.01$, with Buffer A $(\mathrm{pH} 6.0)$ until the $\mathrm{A}_{280 \mathrm{~nm}}<0.01$, with Buffer A (pH 5.3) to elute the monomeric rFGF7-His protein fraction, and finally with Buffer A ( $\mathrm{pH} 4.5)$ to elute the multimeric rFGF7-His protein fraction. Column eluates were monitored at $280 \mathrm{~nm}$ by a Pharmacia Uvicord spectrophotometer connected to analog-to-digital boards of an Intel-based computer workstation running Rainin Dynamax software.

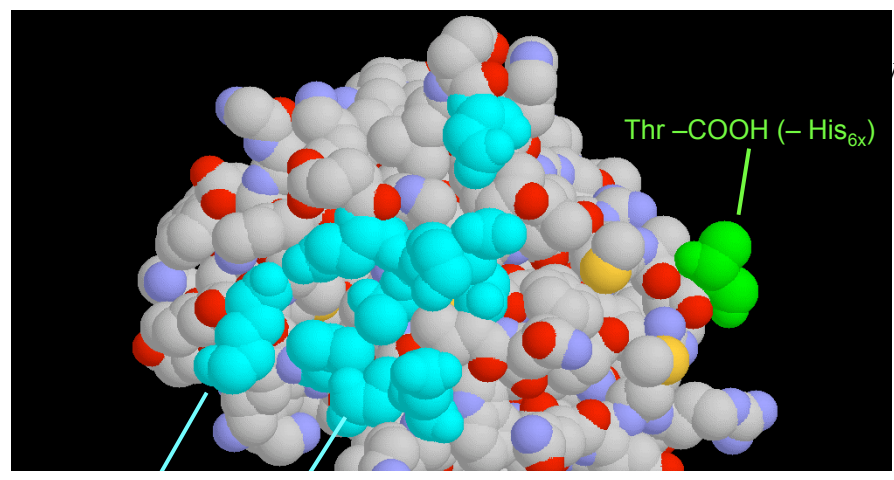

Supplemental Figure 2. Heparinbinding region and $\mathrm{C}$-terminal $\mathrm{His}$ hexamer of rFGF7-His is accessible to the solvent. Shown is a spacefilling model based on the published coordinates for the 3.1 angstrom crystal structure of rat FGF7 (1) (PDB Accession 1QQK). Amino acids identified as comprising 
buffer, at $1 \mathrm{ml} \mathrm{min}^{-1}$. After a baseline was established, the column was developed with step gradients of increasing $\mathrm{LiCl}$ concentrations: $0.5 \mathrm{M}, 1.0 \mathrm{M}, 1.5 \mathrm{M}$, and 2.0 M. rFGF7-His was eluted at $1.0 \mathrm{M} \mathrm{LiCl}$.

\section{Results: Distribution and Characterization of FGF-7 Sequences in the Human Genome}

Supplemental Figure 3 displays a detailed sequence alignment for the duplicated copies that have complete sequence information for exons 3 and 4 . Of interest was which duplicated copies retained coding potential. Exon 4 is divided into the coding and noncoding segments at position 12719 (vertical line within the exon 4 box). Frameshift mutations in exon 4 were detected at 2, 2, and 1 site(s) of the duplicated copies on chromosome 18,21, and the region proximal to the centromere of 9q, respectively. Supplemental Figure 3 also includes the sequence alignment at the junctions of the FGF-7 duplication. The 5' boundaries of the FGF-7 duplicated sequences on chromosome 18, 21, 9pm, 9qt, and 9qc all appeared to be different except that the sequence homology of 9pm and 9qt extends beyond the FGF-7 homology. The sequence homology at the 3' ends of chromosome 18, 21, and 9pm also extends beyond the FGF- 7 homology. This indicates that the FGF-7 duplicated segments on these chromosomes are parts of a larger duplication unit. 


\section{Supplemental Figure 3. Sequence alignment of exons 3 and 4, and of adjacent regions, of the}

FGF-7 gene among human chromosomes. Top line, FGF-7 gene of chromosome 15. Nucleotides that do not match with the FGF-7 gene of chromosome 15 are in black background. Boxed areas, exons 3 and 4. $C h r 9 p c$, FGF-7 copy located proximal to the centromere on the p arm. Chr9pm, middle copy on the p arm. Chr9pt, copy located proximal to the telomere on the p arm. Chr9qt, copy located proximal to the telomere on the $\mathrm{q}$ arm. $C h r 9 q c$, copy located proximal to the centromere on the $\mathrm{q}$ arm. 
chr15 : ACCGGAAGAAACTTGGAATCCCTCGTGACAGTGAAGTTCTGGATAAGAAAGTGAAAGACATGACAGCACAGGTGTTGTTTTCATTTTTTCTTCTAGTTTT

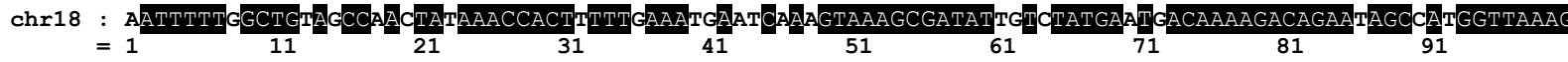

chr15 : ATCTCATGACTTTAGAAGTGAAAAAAAAAAAAAAGCTGTGAAAGTAAACACCTGGCCATTAACAACAGTGTTGAAAATAAGACTTGTCTTTCTGGCTGTT

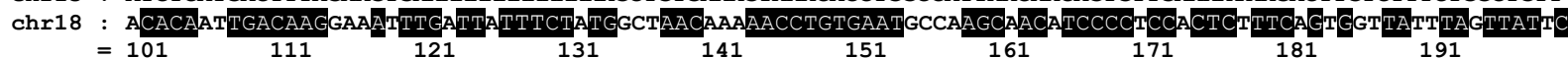

chr15 : AGCTTAGAATAACTGAAAAAATAGGCTCCTGGAGTTTTATGTACATTTTATGAAGAACGCATTTGAAGTAAAGTGAAATTGGGGTGCATTAGTTAAGATT chr18 : AGCTTAGAATAACTGAAAAAATAGGCTCCTGGAGTTTTATGTACATTTTATGAAGAATGCATTTGAAGTAAAATGAAATTGGGGTCATTAGTTAAGATT $\begin{array}{llllllllll}201 & 211 & 221 & 231 & 241 & 251 & 261 & 271 & 281 & 291\end{array}$

After $3,700 \mathrm{bp}$ of intron $2 \ldots$

chr15 : AAGAATTGCTTGATCCTGGGAGGTTCAAGACCCTTGACAATTTGGCCСTAACTCTTTTTCCACCСTCTCACTATTTTGTTACACTTCCTATTCCAGACAT $\operatorname{ch} r 18$ : AAGAACTGCTTGAACCTGGGAGGTTCAAGACCCTTGACAATTTGGCCСTGACTCTTTTTCCACССTCTCACTATTTTGTTACACTTCCTATTCCAGATAT chr21 : ATATACATGCTTCAGATCCAGGATTTATGTCATGGGGATGGATCAGGAGTCCTTGAAACCTGGCACTTATTAAтAAATGTTGTTTCTAATTTTCTATCA

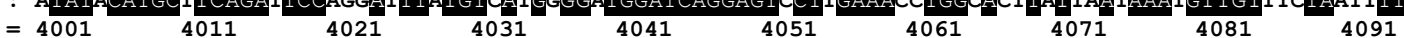

chr15 : ACAGCTCTAGTTTCAATCTTCTGAACATGACTTTTGAATCTTTGCTTCCATGGCCTTGCACATAGTACTTTTATCTCTCAATTAGCATGTTTCCATCTTT chr18 : ACAGCTCTAGTTTCAATCTTCTGAACACGACGTTTGAATCTTTGCTTCCATGGCCTTGCACATAGTACTTTTATCTCTCGATTAGCATGTTTCCATCTTT chr21 : CTGCAAGGTACTCTTATAGACACCATGTGAACATAGTACTTTTATCTCTCTAGCCTTGCACATAGTACTTTTATCTCTCGATTAGCATGTTTCCATCTTI

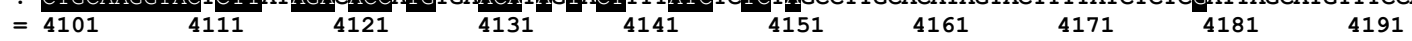

After 600 bp of intron $2 \ldots$

chr15 : TATTATTAAAGTCAGCTGCATTTAAAAATTAGTAGACTTTGTATTTTAGAACACTTTTAGATTTACAAAAAAAATTGAGCAGATAGGCCAGGCGTGGTGG chr18 : TATTATTAAAGTCAGCTGCATTTAAAAATTAGTAGAATTTGTATTTTAGAACACTTTTAGATTTAAAAAAATAATTGAGCAGATAGGCCGGGCGCGGTGG chr21 : TATTATTAAAGTCAGCTGCATTTAAAAATTAGTAGA TTTGTATTTTAGAACACTTTTAGATTTAAAAAAATAATTGAGCAGATAGGCTGGGCATGGTGG chr9pm: CCACAAATCAAAGAACTCAGAGAACACCAGATGCTACTATCCTGTCATAAATTAGGTAGTAMTTTATTAAAATAAAAAGATAGGGCCGGGGCGGTGG chr9qt: CCACAAATCAAAGAACCTCAGAGACACCAGATGCTACTATCCTGTCATAAATTAGGTAGTTATTTTATTAAAATAAAAAGATAGGGCCGGGCGCGTGG

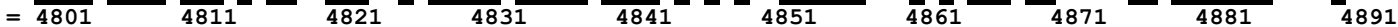

chr15: СTCACGCCTGTAATCCCAGCACTTTGGGAGGCTGAGGTGGGCAGATCACAAGGTCAGGAGATTGAGACCATCCTGGCTAACACGGTGAAACCTCGTCTCT chr18 : СTCACCCCTGTAATCCCAGCACTTTGGGAGGCCGAGGTGGGCAGATCACAAGGTCAGGAGATCGAGACCATCCTGGCTAACACGGTGAAACCTCGTCTCT chr21 : СTCACCCCTGTAATCCCAGCACTTTGGGAGGCGAGGTGGÄCAGATCACAAGGTCAGGAGATCAAGACCATCCTGGTTAACACGGTGAAACCTCGTCTCT chr9pm: CTCACGCCTGTAATCCCAGCACTTTGGGAGGCTGAGGCGGGTGGATCACAAGGTCAGGAGATCGAGGCCATCCTGGCTAACACGGTGAAACCCTGTCTCT chr9qt: СTCACGCCTGTAATCCCAGCACTTTGGGAGGCTGAGGCGGGTGGATCACAAGGTCAGGAGATCGAGGCCATCCTGGCTAACACGGTGAAACCCTGTCTCT $=4901$ 4911 4921 4931 4941 4951 4961 4971 4981 4991

After 800 bp of intron $2 \ldots$

chr15 : AAAACCTGGGGGCATGACTGCTGGATTATATAGTAAGAGTATGTTTAGTTTTTAAGAAACTGCCAAACTGTCTTCCCATGTGACTGCACCATTTTGCATT chr18: AATACCTGGGGGATGACTGCTGGATTATAT----AACATATTCAGTTTTTAAGAAACTGCCAAATTGTCTTCCCATGTGACTGCACCATTTTGCATT chr21 : AATACCTGGGGGCATGACTGCTGGATTATAT-----AGCATATTCAGTTTTTAAGAAGCTGCCAAATTGTCTTCCCATGTGACTGCACCATTTTGCATT chr9pm: AATACCTGGGGGCATGACTGCTGGATTATAT-----AGCATGTTTAGTTTTTAAGAAACTGCCAAACTGTCTTCCCATGTGACTGCACCATTTTGCATT chr9qt: AATACCTGGGGGCATGACTGCTGGATTATAT------AGCATGTTTAGTTTTTAAGAAACTGCCAAACTGTCTTCCCATGTGACTGCACCATTTTGCATT chr9qc: CAACAGAGTCTTTAGTTGACCATAGAGCTCAGCACAGAGGCCTGCTCAAAGGAGAAGTTTGATATACTTTACATGACTTAAATGATTTTAACTTTGTTGA

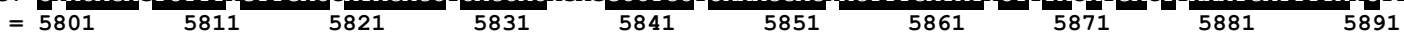

chr15 : CCCACTAGTAATGAAAGAGTTTTGGTTGCTTTGCATCCTTGCCAGCATACAGCACTGTTAGTTTTTTGGAGTTTAGTCACGCTAATAGTTGTACAGAGGT chr18 : CCCACTAGTAATGAAAGAGTTTTGGTTGCTTTGCATCCTTGCCAGCGTACAGCACTGTCAGTTTTCTGGAGTTTAGTCATGCTAATAGTTGTATAGAGGT chr21 : CCCACTAGTAATGAAAGAATTTTGGTTGCTTTGCATCCTTGCCAGCATACAGCACTGTCAGTTTTTTGGAGTTTAGTCATGCTAATAGTTGTATAGAGGT chr9pm: CCCACTAGTAATGAAAGAGTTTTGGTTGCTTCGCATCCTTGCCAGCATACAGCACTGTCAGTTTTTTGGAGTTTAGTCATGCTAATAGTTGTATAGAGGT chr9qt: CCCACTAGTAATGAAAGAGTTTTGGTTGCTTCGCATCCTTGCCAGCATACAGCACTGTCAGTTTTTTGGAGTTTAGTCATGCTAATAGTTGTATAGAGGT chr9qc: AACTTGTTTAATGAAAGAGTTTTGGTTGCTTTGCATCCTTGCCAGCATACAGCACTGTCAGTTTTTTGGAGTTTAGTCATGCTAATAGTTGTATAGAGGT

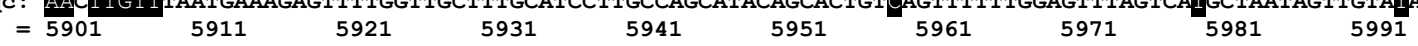

After 5,300 bp of intron $2 \ldots$

EXON 3

chr15 : TTTCTGTGATTCCTTGCAGATATCATGGAAATCAGGACAGTGGCAGTTGGAATTGTGGCAATCAAAGGGGTGGAAAGTGAATTCTATCTTGCAATGAACA chr18 : TTTCTGTGATTCCTTGAATATATCATGGAAATCAGGACAGTGGCAGTTGGGATTGTGGCAATCAAAGGGGTGGAAAGTGAATTCTATCTTGCAATGAAT chr21 : TTTCTGTGATTCCTTGCAGATATCATGGAAATCAGGACAGTGGCAGTTGGGATTGTGGCAATCAAAGGGGTGGAAAGTGAATTCTATCTTGCAATGAAT chr9pm: TTTCTGTGATTCCTTGCAGATATCATGGAAATCAGGACAGTGGCAGTTCGGATTGTGGCAATCAAAGGGGTGGAAAGTGAATTCTATCTTGCAATGAAC chr9qt: TTTCTGTGATTCCTTGCAGATATCATGGAAATCAGGACAGTGGCAGTTCGGATTGTGGCAATCAAAGGGGTGGAAAGTGAATTCTATCTTGCAATGAAC chr9qc: TTTCTGTGATTCCTTGCAGATATCATGGAAATCAGGACAGTAGCAGTTGGGATTGTGGCAATCAAAGGGGTGGAAAGTGAATTCTATCTTGCAATGAAC

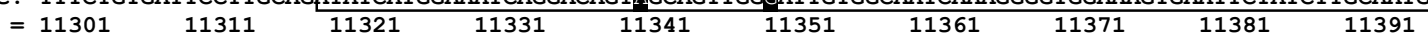


EXON 3

chr15: AGGAAGGAAAACTCTATGCAAAG $\operatorname{chr18:~AGGAAGGAAAACTCTACGGAAAGFTATTGATATTGATCGCTTAGGCTTAATTTTTAAAACTCATTTTTGTCGAAATATCTCACCATTCTGAAAAGGAA~}$ chr21: AGGAAGGAAAACTCTATGCAAAG\&TATTGATAATTGATCGCTTAGGCTTAATTTTTAAAACTCATTTTTGTCAAAATATCTCACCATTCTGAAAAGGAAA chr9pm: AGGAAGGAAAACTCTATGCAAAG\&TATTGATAATTGATAGCTTAGGCTTAATTTTTAAAACTCATTTTTGTTGAAATATCTCACCATTCTGAAAAGTAAA chr9qt: AGGAAGGAAAACTCTATGCAAAG\&TATTGATAATTGATAGCTTAGGCTTAATTTTTAAAACTCATTTTTGTTGAAATATCTCACCATTCTGAAAAGTAAA chr9qc: AGGAAGGAAAACTCTATGCAAAGGTATTGATAATTGATAGCTTAGGCTTAATTTTTAAAACTC--TTTTGTTGAAATATCTCACCATTCTGAAAAGTAAA

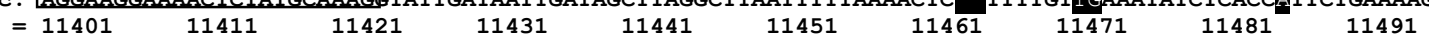

After $1,000 \mathrm{bp}$ of intron $3 \ldots$

EXON 4

chr15 : GTTTGTTTGTTTGTTTTAACAGAAAGAATGCAATGAAGATTGTAACTTCAAAGAACTAATTCTGGAAAACCATTACAACACATATGCATCAGCTAAATGG chr18 : GTTTGTTTGTTTGTTTTAACAGAAAGAATGCAATGAAGATTGTAACTTCAAAGGACTAATTCTGGAAAACCATTACAACACATATGCCTCAGCTAAATGG chr21 : GTTTGTTTGTTTGTTTTAACAGAAAGAATGCAATGAAGATTGTAACTTCAAAGGACTAATTCTGGAAAACCATTACAACACATATGCCTCAGCTAAATGG chr9pm: GTTTGTTTGTTTGTTTGAACAGAAAGAATGCAATGAAGATTGTAACTTCAAAGAACTAATTCTGGAAAACCATTACAACACATATGCAGCAGCTAAATGG chr9qt: GTTTGTTTGTTTGTTTGAACAGAAAGAATGCAATGAAGATTGTAACTTCAAAGAACTAATTCTGGAAAACCATTACAACACATATGCAGCAGCTAAATGG chr9qc: GTTTGTTTGTTTGTTTAACAGAAGGAATGCAATGAAGATTGTAACTTCAAAGATCTAATTCTGGAAAACCATTACAACACATATGCAGCAGCTAAATGG

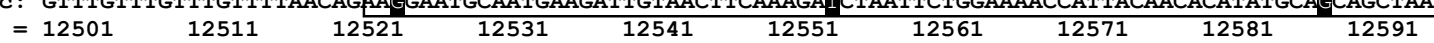

EXON 4

chr15: ACACACAACGGAGGGGAATGTTTGTTGCCTTAAATCAAAAGGGGATTCCTGTAAGAGG-AAAAAAAACGAAGAAAGAACAAAAAACAGCCCACTTTCTT chr18 : ACACACAATGGAGGGGAAATGTTTGTTGCCTTAAATCAAAAGGGGATTCCTCTAAGAGG-AAAAAAAATGAAGAAAGAAC-AAAAACAGCCCACTTTCTT chr21: ACACACAATGGAGGGGAATGTTTGTTGCCTTAAATCAAAAGGGGATTCCTGTAAG--G-AAAAAAAATGAAGAAAGAAC-AAAAACAGCCCACTTTGTT chr9pm: ACACACAATGGAGGGGAAATGTTTGTTGCCTTAAATCAAAAGGGGATTCCTGTAAGAGG-AAAAAAAACGAAGAAAGAACAAAAAACAGCCCACTTTCTT chr9qt: ACACACAATGGAGGGAAATGTTTGTTGCCTTAAATCAAAAGGGGATTCCTGTAAGAGG-AAAAAAAACGAAGAAAGAACAAAAAACAGCCCACTTTCTT chr9qc: ACAAACAACGGAGGGGAATGTTTGTGGCCTTAAATCAAAAGGGGATTCCTGTAAGAGGAAAAAAAAACAAAGAAAGAACAAAAAACAGCCCACTTTCTT

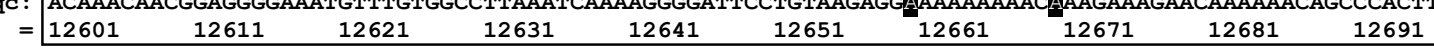

END (of coding) EXON 4

chr15: CCTATGGCAATAACTTAA PTGCATATGGTATATAAAGAACCAGTTCCAGCAGGGAGATTTCTTTAAGTGGACTGTTTTCTTTCTTCTCAAAATTTTCTTT chr18: CCTATGGCAATAACTTAA PTGTATATGGTATATAAAGAACCAGTTCCAGCAGGGAGATTCTTTAAGTGGACTGT----TTTCTTCTCAAAATTTTCTTT chr21 : CCTATGGCAATAACTTAA ITGCATATGGTATATAAAGAACCAGTTCCAGCAGGGAGATTTCTTTAAGTGGACTGTTTTCTTTCTTCTCAAAATTTTCTTT chr9pm: CCTATGGCAATAACTTAA PTGCATATGGTATATAAAGAACCAGTTCCAGTAGGGAGATTTCTTTAAGTGGACTGTTTTCTTTCTTCTCAAAATTTTCTTT chr9qt: CCTATGGCAATAACTTAA PTGCATATGGTATATAAAGAACCAGTTCCAGTAGGGAGATTTCTTTAAGTGGACTGTTTTCTTTCTTCTCAAAATTTTCTTT chr9qc: CCTATGGCAATAACTTAA PTGCATATGGTATATAAAGAACCAGTTCCAGCAGGGAGATTTCTTTAAGTGGACTGTTTTCTTTCTTCTCAAAATTTTCTTT

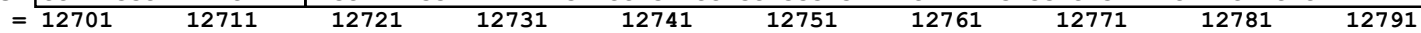

After 2,700 bp of $3^{\prime}$ UTR ..

$$
\text { EXON } 4
$$

chr15: AAAATGTTCTTTGAAAGATAAAATTAAATACATGAGTTTCTAACAATTA\&AAAAGAAAAAATTAAAACATGAAATGATAACAAAAGTAAACAAAAGATAC $\mathrm{ch} r 18:$ AAAATGTTCTTTGAAAGATAAAATTAAATACATGAGTTTCTAACAATTAGAAAGGAAAAAATTAAAATATGAAATGATAACAAAAGTAAACAGAAGATAC chr21: AAAATGTTATTGAAAGATAAAATCAAATACATGAGTTTCTAACAATTAGAAAGGAAAAAATTAAAATATGAAATGATAACAAAAGTAAACA GAAGATAC chr9pm: AAAATGTTCTTTGAAAGATAAAATTAAATACATGAGTTTCTAACAATTAGAAAGGAAAAAATTAAAATATGAAATGATAACAAAAGTAAACAAAAGATAC chr9qt: AAAATGTTCTTTGAAAGATAAAATTAAATACATGAGTTTCTAACAATTAGAAAGGAAAAAATTAAAATATGAAATGATAACAAAAGTAAACAAAAGATAC chr9qc: AAAAATTTCTTTGA---AAGATAAAATACATGAGTTCTAACAATTAGAAAGGAAAAAATGAAAATATGAA---ATAACAAAAGTAAACAAAAGATAC

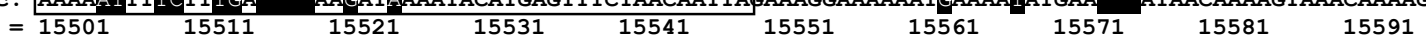

After $100 \mathrm{bp} \ldots$

chr15 : TACAAATTCAATTTAATGACAGAAGAGAAGGGATGCTGGAGG----TAAATTCTTAGGGTTTCTATCTCATAGAGTTTGCTCTTCTGGTTCTCTAGACTG chr18 : TACAAATTCAATTTAATGACAGAAGAGAAGGGATGCTGGAGG----TAAATTCTTAGGGTTTCTATCTCATAGAGTTTGCTCTTCTGGTTCTCTAGACTG chr21 : TACAAATTCAATTTAATGACAGAAGAGAAGGGATGCTGGAGG----TAAATTCTTAGGGTTTCTATCTCATAGAGTTTGCTCTTCTGGTTCTCTAGACTG chr9pm: TATGAATTCAATTTAATGACAGAAGAGAAGGGATGCTGGAGG----TAAATTCTTAGA GTTTCTATCTCATAGAGTTTGCTCTTCTGATTCTCTAGACTG chr9qt: TATGAATTCNNNNNNNNNNNNNNNNNNNNNNNNNNNNNNNNNNNNNNNNNNNNNNNNNNNNNNNNNNNNNNNNNNNNNNNNNNNNNNNNNNNNNNNNNNN chr9qc: САCAAATTCAATTTAATGAAAGAAGAGAAGGGATGCTGGAGGTAAATAAATTCTTAGAGTTTCTATCTCATAGAGTTTGCTCTTCTGGTTCTCTAGACTG

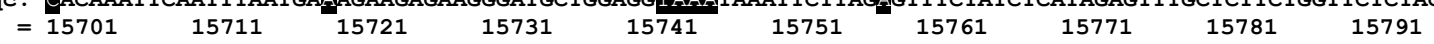

After 700 bp ...

chr15 : GCTACTCTTTATTCCACTTCTGTACAGTATTTATTCAACCAAGCTGCTGCTTTCAATGAAGGTCACTTGTTCCTTCAGGGACACATATACTCCCACCTAT chr18 : GCTACTCTTTATTCCACTTCTGTACAGTATTTATTCAACCAAGCTGCTGCTTTCAATGAAGGTCACTTGTTCCTTCAGGGACACATATACTCCCACCTAT chr21 : GCTACTCTTTATTCCACTTCTGTACAGTATTTATTCAACCAAGCTGCTGCTTTCAGTGAAGGTCACTTGTTCCTTCAGGGACACATATACTCCCACCTAT chr9pm: GCTACTCTTTATTCCACTTCTGTACAGTATTTATTCAACCAAGCTGCTGCTTTCAATGAAGGTCACTTGTTCCTTCAGGGACACATGTACTCCCACCTAT chr9qc: GCTACTCTTTATTCCACTTCTATACAGATCATGTCATCTTCAAATAGAGACACTTTCACTTCTTTTCCAATTTGGATGCCTTTTATTTCTTTTTCTTACC $=16501$ 16511 16521 16531 16541 16551 16561 16571 16581 16591

After $1,900 \mathrm{bp} \ldots$

chr15: TGTTTTTGCATCAGAATGATTATGGAATACAAAGTGCACAAAAGTTCTCCCTCCCCCACCACAGTCCATTCTCAAACAGAGCAGCCAAAGTGATTCTGTT chr18: TGTTTTTGCATCAGAATGATTATGGAATAGAAAAAAGCATTTCATGCAAATGGACACCAAA GCGAGCAGCAGTAGCTATTCTCATATGAGACAAAACA chr21: TGTTTTTGCATCAGAATGATTATGGAACAGA

chr9pm: TGTTTTTGCATCAGAATGATTATGGAATAGA $=18501$ 18511 18521 18531 